\title{
The Moment Map: Nonlinear Dynamics of Density Evolution via a Few Moments*
}

\author{
D. Barkley ${ }^{\dagger}$, I. G. Kevrekidis ${ }^{\ddagger}$, and A. M. Stuart ${ }^{\dagger}$
}

\begin{abstract}
We explore situations in which certain stochastic and high-dimensional deterministic systems behave effectively as low-dimensional dynamical systems. We define and study moment maps, maps on spaces of low-order moments of evolving distributions, as a means of understanding equation-free multiscale algorithms for these systems. The moment map itself is deterministic and attempts to capture the implied probability distribution of the dynamics. By choosing situations where the lowdimensional dynamics can be understood a priori, we evaluate the moment map. Despite requiring the evolution of an ensemble to define the map, this can be an efficient numerical tool, as the map opens up the possibility of bifurcation analyses and other high level tasks being performed on the system. We demonstrate how nonlinearity arises in these maps and how this results in the stabilization of metastable states. Examples are shown for a hierarchy of models, ranging from simple stochastic differential equations to molecular dynamics simulations of a particle in contact with a heat bath.
\end{abstract}

Key words. equation-free, multiscale, moment map, metastable states

AMS subject classifications. 65C30, 34F $05,60 \mathrm{H} 10,60 \mathrm{H} 35$

DOI. $10.1137 / 050638667$

1. Introduction. An equation-free framework has recently been developed as a means of computationally analyzing the dynamical behavior of a large class of complex multiscale dynamical systems. The systems may be either stochastic or deterministic with many degrees of freedom and subject to random initial data. The key observation behind the equationfree framework is that in many cases the quantities of interest are averages or low-order moments of evolving distributions, which are smooth in space and time and which evolve effectively as closed low-dimensional systems. In effect, the low-order moments evolve as though they are governed by reduced closed equations, even though the reduced equations are not analytically available. Algorithms for performing scientific computing tasks such as numerical integration or bifurcation and stability analysis of these unavailable reduced equations have been developed (e.g., coarse projective integration, coarse Newton-GMRES; see $[40,12,25,26]$ and references therein). These algorithms are based on traditional continuum numerical analysis, "wrapped" around direct fine-scale simulation. The purpose of this paper is to establish a mathematical framework for understanding the behavior of coarse dynamics and coarse bifurcation methods on problems which exhibit metastable behavior.

\footnotetext{
${ }^{*}$ Received by the editors August 23, 2005; accepted for publication (in revised form) by T. Kaper February 27, 2006; published electronically July 17, 2006.

http://www.siam.org/journals/siads/5-3/63866.html

${ }^{\dagger}$ Mathematics Institute, University of Warwick, Coventry, CV4 7AL United Kingdom (barkley@maths.warwick. ac.uk, stuart@maths.warwick.ac.uk).

${ }^{\ddagger}$ Department of Chemical Engineering, Program in Applied and Computational Mathematics, and Mathematics, Princeton University, Princeton, NJ 08544 (yannis@Princeton.edu).
} 


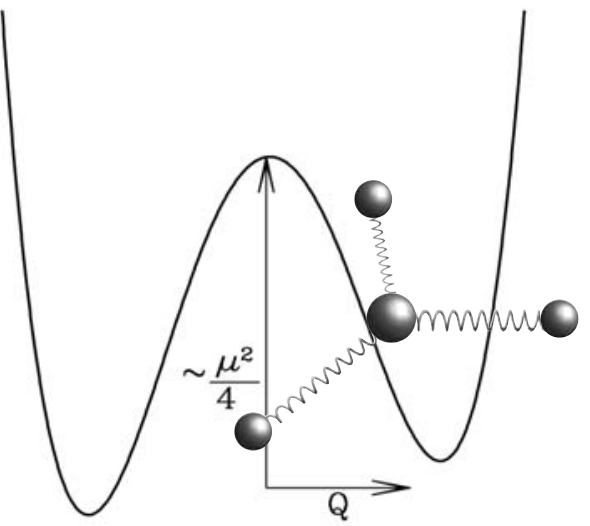

Figure 1. Model system. A distinguished particle (light gray) sits in an asymmetric double well potential. The particle is coupled via linear springs to $N$ other (bath) particles (of which three are shown as an illustration). The full Hamiltonian system has $N+1$ degrees of freedom.

The systems we consider are exemplified by the following model. A particle, called the distinguished particle, with position $Q$ and momentum $P$, sits in a potential well $V(Q)$. It is coupled via linear springs to a large number $N$ of particles comprising a heat bath; see Figure 1. The potential well considered here is a slightly asymmetric double well. The full system is an $N+1$ degree-of-freedom Hamiltonian system. A detailed description of the model, including the choice of spring constants, masses, and initial data for the bath particles is given below and in $[10,28,43]$. The important point is that the dynamics of this simple model (and the others that we consider in this paper) is typical of many more complex molecular and stochastic systems in which the state is primarily confined to a few conformations (here defined by the minima of $V$ ) with rare switching events between them. This is illustrated in Figure 2 with a typical trajectory and time series for the distinguished particle.

Consider now the dynamics of an ensemble of trajectories for the model system. Figure 3 illustrates the evolution of an ensemble of trajectories all with the same initial conditions $(Q, P)=(0,3)$ for the distinguished particle, but with different initial data for the bath particles. (The total initial energy of the bath is approximately the same for all realizations; see section 2.) Over a time of order 10 the initial density evolves to a nearly Gaussian density centered near the bottom of the right well, where it remains roughly constant for some time. While not immediately evident, during the initial 10 time units the density is never very far from Gaussian. However, it is evident from the left-hand plot that a small percentage of the realizations are located in the left well at time 10. Over a much longer time scale, $O\left(10^{4}\right)$, the density evolves to the bimodal equilibrium distribution and is hence far from Gaussian. Thus the system exhibits metastability with near equilibration within one well dominating over medium time scales, before the system ultimately converges to an equilibrium distribution which sees both wells. The time it takes the system to reach equilibrium clearly depends on the potential barrier height; the time scale of the intermediate time evolution to the well bottom depends only on properties of the particular well.

Our aim is to study the behavior of coarse dynamics and coarse bifurcation methods on problems which exhibit metastable behavior of this type. In section 2 we introduce a hierarchy 

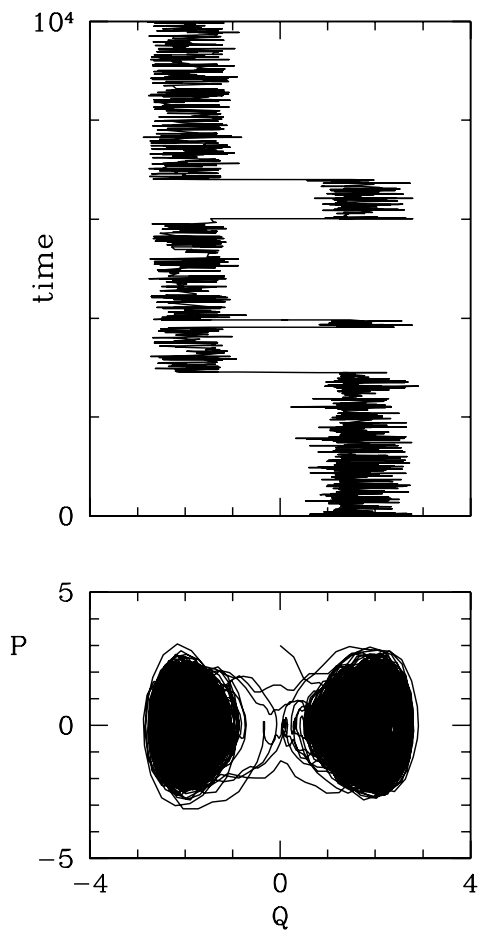

Figure 2. Typical behavior for the dynamics of the distinguished particle in contact with a bath with $N=8000$ particles. Most of the time the distinguished particle is located in one of the two potential wells, but occasionally it makes a jump between wells.

of model problems, all of which exhibit rare transitions between a small number of states, and which we then use throughout as illustrations.

In section 3 we define the discrete-time moment map $\Phi$ for the first $k$ moments of the ensemble of solutions to a time evolving system. Specifically, $\Phi$ will be a deterministic lowdimensional map defined by the short-time evolution of only the low-order moments, in a general setting which applies to systems of both ordinary differential equations (ODEs), with randomness from initial data, and stochastic differential equations (SDEs), with randomness from initial data and Brownian driving noise. Figure 4 illustrates the first-order moment map for the heat bath example. The short-term dynamics of the map resemble those of the ensemble, but significantly, the moment map has stable fixed points corresponding to means of metastable densities centered in each well. The second-order moment map (not illustrated) additionally captures the widths of the metastable measures. The algorithms we study are based on these maps. The maps, and their fixed points, are good approximations on time scales on which the evolving probability density function is well represented by the number of moments used.

The heart of this paper is section 4, which is devoted to the study of the moment map when applied to a variety of model systems. We use a combination of exact solutions for Gaussian problems, approximate solutions for metastable systems, and numerical experiments. Of central interest is the observation that the moment maps stabilize the metastable states in 

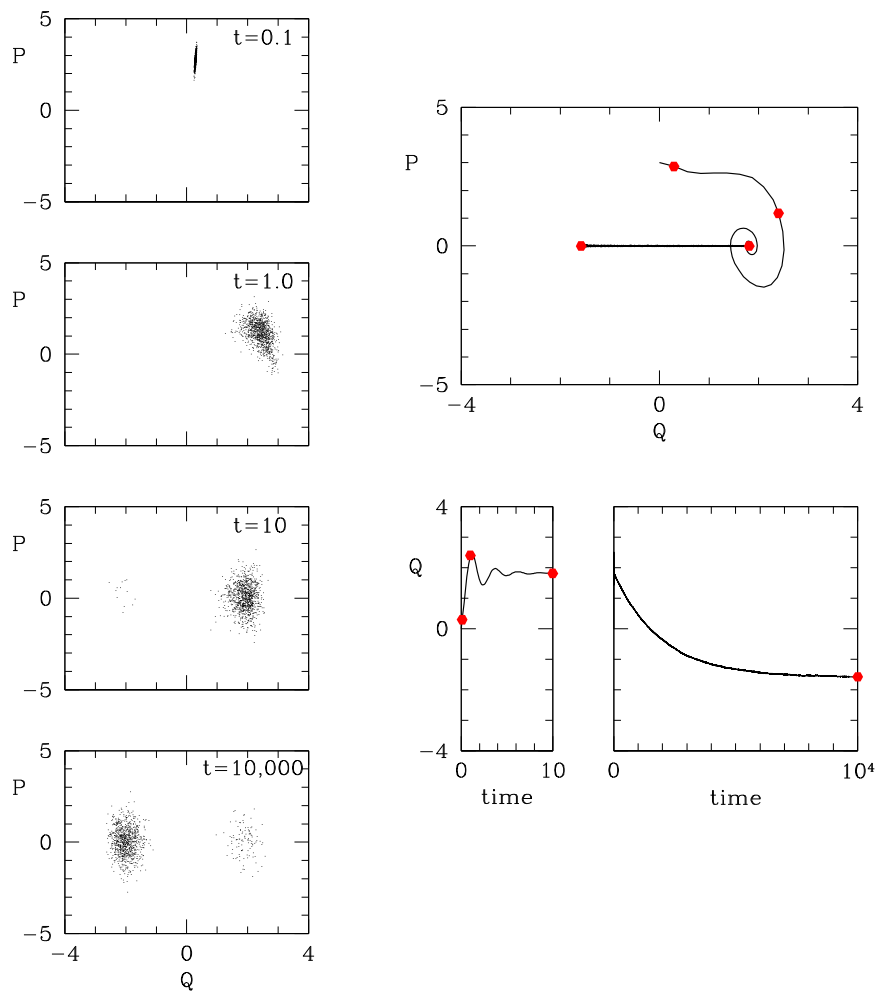

Figure 3. Evolution of an ensemble of $10^{4}$ realizations for the model system. On the left, density plots of position and momentum of the distinguished particle are shown at four times as labeled. For clarity only $10^{3}$ realizations are plotted. The initial conditions are $(Q, P)=(0,3)$. The right shows the trajectory and time series for the ensemble expectations. Red points indicate the four times shown on the left. There are $N=8000$ particles in the heat bath.

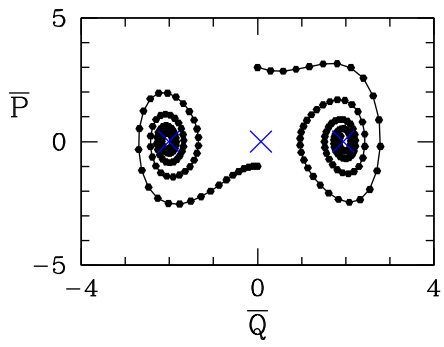

Figure 4. Dynamics of a moment map for the first-order moments of the position $Q$ and momentum $P$ of the distinguished particle. The map has three fixed points, two stable foci and one unstable saddle, shown as blue crosses. Two trajectories are shown, one evolving to each of the stable fixed points. The right trajectory has initial condition $(\bar{Q}, \bar{P})=(0,3)$, corresponding to that in Figure 3 . There are $N=8000$ particles in the heat bath.

the model problems of interest. The moment map is a nonlinear map, defined from the linear flow of probability densities for ODEs and SDEs, with the nonlinearity entering through the process of repeatedly projecting onto the space of moments. This process of nonlinearization 
creates interesting fixed points that are associated with metastable behavior and are amenable to low-dimensional bifurcation analyses; related issues are addressed in [5, 20]. Section 5 contains our concluding remarks.

2. Model problems. We consider three example systems in this paper. Two are SDEs, and one is the ODE heat bath model described in the introduction and illustrated in Figure 1. A major thrust of this paper is to establish, through computational experimentation, that the moment map stabilizes metastable behavior arising from the slow dynamics between potential wells with large energy barriers; this gives rise to nonlinear phenomena, such as bifurcations, in the moment map. Such phenomena can be illustrated both in SDE models and in the ODE heat bath model. Furthermore, in various parameter limits, the SDEs can be derived as approximations for the heat bath, further justifying their study.

2.1. Heat-bath model. This model problem is defined by the Hamiltonian

$$
H(Q, P, q, p)=\frac{1}{2 M} P^{2}+V(Q)+\sum_{j=1}^{N} \frac{p_{j}^{2}}{2 m_{j}}+\sum_{j=1}^{N} \frac{k_{j}}{2}\left(q_{j}-Q\right)^{2},
$$

where $Q$ and $P$ are the position and momentum of a distinguished particle of unit mass in a potential field $V(\cdot)$. The $q_{j}$ 's and $p_{j}$ 's are the coordinates and momenta of $N$ particles that are referred to as heat bath particles. The $j$ th heat bath particle has mass $m_{j}$ and interacts with the distinguished particle via a linear spring with stiffness constant $k_{j}$. If the distinguished particle were held fixed, it would be the anchor point of $N$ independent oscillators with frequencies $\omega_{j}=\left(k_{j} / m_{j}\right)^{1 / 2}$. The numerical experiments are all conducted with mass $M=1$.

Initial conditions for the distinguished particle are $Q(0)=Q_{0}, P(0)=P_{0}$. The initial data for the heat bath particles, $q_{j}(0)=q_{j}^{0}$ and $p_{j}(0)=p_{j}^{0}$, are randomly drawn from a Gibbs distribution with inverse temperature $\beta$. The Gibbs measure is conditioned by the (nonrandom) initial data $Q_{0}$ and $P_{0}$. For fixed $Q, P$ the Hamiltonian (2.1) is quadratic in $q, p$, and hence the corresponding measure is Gaussian. It is easily verified that

$$
\begin{aligned}
& q_{j}^{0}=Q_{0}+\left(1 / \beta k_{j}\right)^{1 / 2} \xi_{j}, \\
& p_{j}^{0}=\left(m_{j} / \beta\right)^{1 / 2} \eta_{j},
\end{aligned}
$$

where $\xi_{j}, \eta_{j} \sim \mathcal{N}(0,1)$ are mutually independent sequences of independently and identically distributed (i.i.d.) random variables.

This leaves the specification of the values for $k_{j}$ and $m_{j}$. For our purposes the only important property is that the frequencies $\omega_{j}=\left(k_{j} / m_{j}\right)^{1 / 2}$ cover an increasingly large range in an increasingly dense manner as the number of particles $N$ increases. Hence what we actually specify is the frequencies. These are chosen to be random and uniformly distributed in $\left[0, N^{1 / 3}\right]$,

$$
\omega_{j}=N^{1 / 3} \nu_{j}, \quad \nu_{j} \text { i.i.d., } \quad \nu_{1} \sim \mathcal{U}[0,1] .
$$

It is important to note that, in addition to the initial data, the model specification itself contains this random element. We shall be careful to distinguish between the two types of randomness. 
From the frequencies the spring constants and masses are given by

$$
k_{j}=\frac{2 \alpha^{2}}{\pi\left(\alpha^{2}+\omega_{j}^{2}\right)} \frac{N^{1 / 3}}{N}, \quad m_{j}=\frac{k_{j}}{\omega_{j}^{2}}
$$

with $\alpha>0$. See [28] for further details. The parameters $\alpha$ and $\beta$ are fixed at $\alpha=100, \beta=2$.

The potential considered in this paper is

$$
V(Q)=\frac{Q^{4}}{4}-\frac{\mu Q^{2}}{2}+\nu Q
$$

where $\mu$ and $\nu$ are parameters with $\nu$ typically small.

2.2. Two-dimensional SDE approximation. For large $N$ and $\alpha$, the distinguished particle $Q$ in the heat bath model can be approximated by the SDE

$$
M \ddot{Q}+\gamma \dot{Q}+V^{\prime}(Q)=\sqrt{2 \gamma / \beta} \dot{W} .
$$

A theorem justifying this approximation can be proved using the techniques of weak convergence, by taking the limit $N \rightarrow \infty$ (see [28]) and then $\alpha \rightarrow \infty$ (see [34]). In the absence of noise (the zero temperature limit $\beta \rightarrow \infty$ ) this damped Hamiltonian system exhibits decay towards stationary points with zero velocity and positions at the critical points of $V$. The presence of noise (finite $\beta$ ) then induces transitions between the minima of $V$, with time scales determined by the well-depths relative to the size of the noise.

2.3. One-dimensional SDE approximation. The stochastic dynamics between potential wells is also present in simple one-dimensional SDEs. A particular instance of such a onedimensional SDE follows from (2.4) for $M \ll 1$. In the limit $M \rightarrow 0$, the solutions of (2.4) converge strongly [33] or weakly [11] to solutions of the SDE

$$
\gamma \dot{Q}+V^{\prime}(Q)=\sqrt{2 \gamma / \beta} \dot{W} .
$$

We will use this problem to illustrate the moment map and its properties on systems exhibiting metastable dynamics within potential wells.

2.4. Remark. In most respects the SDE systems derived above are simpler to treat, and computationally they are far less expensive to simulate, than the full heat-bath model. Therefore, when we later use these models as examples, we will study the models in the opposite order from what has just been presented. We start with the one-dimensional SDE and examine its behavior extensively, and then consider more briefly the two-dimensional SDE and the heat bath system of ODEs.

3. The moment map. The central objects of our study are maps on moments. The basic ingredients are an evolution equation (either an SDE or a system of ODEs), a space of loworder moments, and a measure determined uniquely by low-order moments. We refer to the latter as lifting, and the choice of the lifting operator is an essential ingredient in the method. We start with the SDE case, then describe the situation for ODEs. For both the SDE and the ODEs the flow on probability densities is linear. The nonlinearity inherent in the moment map comes from the relationship between the probability density function and its moments. After describing the moment map for the SDE and the ODE, the section concludes with some general remarks. 
3.1. The SDE case. Let $x \in C\left([0, \infty), \mathbb{R}^{d}\right)$ solve the following Itô SDE, driven by Brownian motion $W \in C\left([0, \infty), \mathbb{R}^{m}\right)$ :

$$
\frac{d x}{d t}=f(x)+\sigma(x) \frac{d W}{d t} .
$$

This includes (2.4) and (2.5) as special cases. We will consider ensembles of solutions of this equation, with ensembles taken over multiple driving noises and random initial data. Let $X_{j}(t)$ be the $j$ th moment of $x(t)$, with expectation taken with respect to both the driving Brownian motion $W$ and random initial data, the latter being assumed independent of the Brownian motion. Denote the first $k$ moments of $x$ by $X(t)=\left(X_{1}(t), \ldots, X_{k}(t)\right)$.

Let $\mu$ be a measure on $\mathbb{R}^{d}$ determined by exactly $k$ moments, with density $\hat{\rho}(x ; X)$. Here $X$ denotes the dependence of the density on the $k$ moments, and we require that the $k$ moments of $\hat{\rho}$ are exactly those given by $X$.

We now define the map $\Phi$ on $k$ moments. Fix a time $\tau \in(0, \infty)$ and set

$$
\Phi(X)=X(t=\tau),
$$

where the initial data $X(0)=x_{0}$ is distributed with density $\hat{\rho}(x ; X)$. (Note that if $\tau=0$, then $\Phi$ is the identity.) We refer to this as the moment map. Three choices that need to be made when calculating the moment map are:

(i) the evolution time $\tau$,

(ii) the number of moments we choose to use,

(iii) the lifting step: the way we choose to distribute the initial density based on the moments.

We will return to this dependence in more detail below.

The two examples of the measures which will be used throughout this paper are the Dirac measure and the Gaussian measure, uniquely determined by the first moment and the first two moments, respectively. We emphasize that the moment map is a deterministic map.

In practice the moment map must be obtained numerically, through Monte Carlo simulation, for example. However, it is insightful to describe the definition of the map through the Fokker-Planck equation for (3.1). This linear PDE for the probability densities $\rho(x, t)$ propagated by (3.1) is

$$
\begin{aligned}
\frac{\partial \rho}{\partial t}=-\nabla \cdot(f \rho)+\frac{1}{2} \nabla \cdot \nabla \cdot(\Sigma \rho) & :=\mathcal{L}^{*} \rho, \\
\rho(x, 0) & =\hat{\rho}(x ; X),
\end{aligned}
$$

where $\Sigma=\sigma(x) \sigma(x)^{T}$. Here $\mathcal{L}^{*}$ is the adjoint of the generator for the process $\mathcal{L}$. We denote the solution by

$$
\rho(x, t)=e^{\mathcal{L}^{*} t} \hat{\rho}(x ; X) .
$$

From $\rho(x, t=\tau)$ we can construct $\Phi(X)$ by (3.2). In general the moment map is nonlinear because of the nonlinear dependence of $\hat{\rho}(x ; X)$ on $X$ and the nonlinearity of the map from $\rho(x, \tau)$ to $X(\tau)$. Thus we have constructed a nonlinear map on $\mathbb{R}^{d}$ from the linear flow on $\mathbb{R}^{d}$-valued densities. 


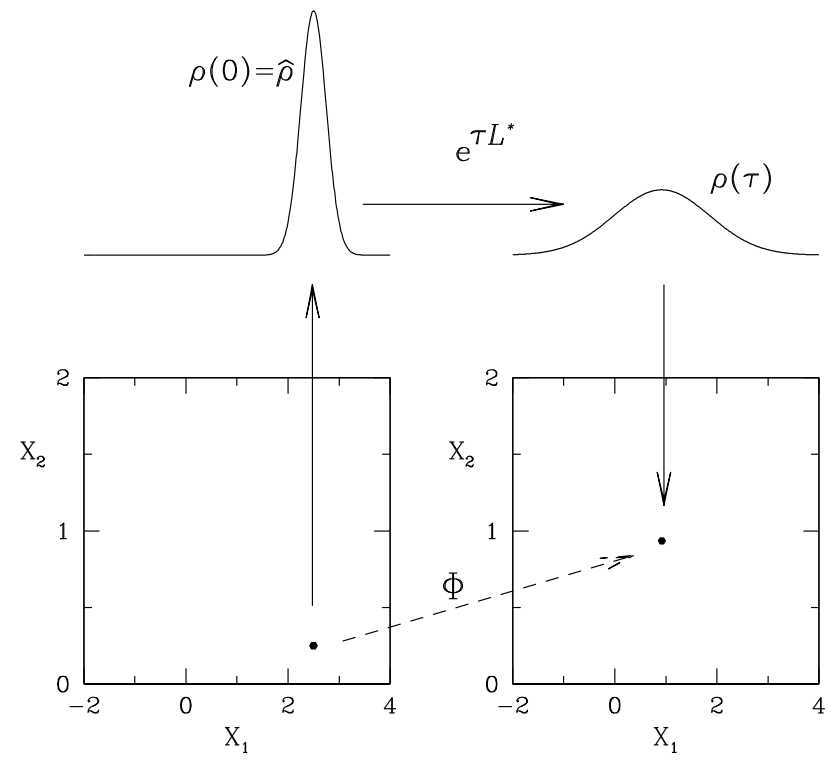

Figure 5. Sketch illustrating the definition of the moment map. From a point $\left(X_{1}, X_{2}\right)$ in moment space a density $\hat{\rho}$ is uniquely determined. This initial density is evolved by the system dynamics (e.g., the Fokker-Planck equation). From the resulting density $\rho(\tau), \tau$ time units later, a new point in moment space is determined. This point is defined to be the image of $\left(X_{1}, X_{2}\right)$ under the moment map.

It is helpful to consider Figure 5, illustrating the definition of the moment map. One should view the map as a composition of three steps: (1) lifting from a space of moments (subset of $\mathbb{R}^{d}$ ) to a space of probability densities (captured by our choice of $\hat{\rho}(\cdot ; X)$ ), (2) time evolution of the density by the underlying process (a linear flow, given by the map $e^{\mathcal{L}^{*} \tau}$ ), (3) projection back to moment space by integrating against the time-evolved measure.

In the case of a single moment, in this paper, we take $\mu$ to be a Dirac measure at $X$, and then $X(\tau)=\mathbb{E} x(\tau)$ can be calculated from

$$
\Phi(X)=\int_{\mathbb{R}^{d}} x e^{\mathcal{L}^{*} \tau} \delta(x-X) d x
$$

In the case of two moments we have

$$
X(\tau)=\left\{\mathbb{E} x(\tau), \mathbb{E}[x(\tau)-\mathbb{E} x(\tau)][x(\tau)-\mathbb{E} x(\tau)]^{T}\right\}
$$

and we take $\mu$ to be a Gaussian measure with mean and covariance determined by these moments. It is convenient to express the moment map in terms of the mean $\bar{x} \in \mathbb{R}^{d}$ and covariance matrix $\Sigma \in \mathbb{R}^{d \times d}$. We obtain the map

$$
\Phi(\bar{x}, \Sigma)=\left\{\begin{array}{l}
\Phi_{1}(\bar{x}, \Sigma) \\
\Phi_{2}(\bar{x}, \Sigma)
\end{array}\right\}
$$


The functions $\Phi_{i}$ are defined as follows. Since the density $\hat{\rho}$ is determined by the moments we are carrying, in this Gaussian case it is

$$
\hat{\rho}(x ; \bar{x}, \Sigma)=\frac{\exp \left(-\frac{1}{2}\left\|\Sigma^{-\frac{1}{2}}(x-\bar{x})\right\|^{2}\right)}{\left.\sqrt{(}(2 \pi)^{d} \operatorname{det} \Sigma\right)} .
$$

Then $\Phi_{1}: \mathbb{R}^{d} \times \mathbb{R}^{d \times d} \rightarrow \mathbb{R}^{d}$ is given by

$$
\Phi_{1}(\bar{x}, \Sigma)=\int_{\mathbb{R}^{d}} x\left\{e^{\mathcal{L}^{*} \tau} \hat{\rho}(x ; \bar{x}, \Sigma)\right\} d x
$$

and $\Phi_{2}: \mathbb{R}^{d} \times \mathbb{R}^{d \times d} \rightarrow \mathbb{R}^{d \times d}$ is given by

$$
\Phi_{2}(\bar{x}, \Sigma)=\int_{\mathbb{R}^{d}}\left(x-\Phi_{1}(\bar{x}, \Sigma)\right)\left(x-\Phi_{1}(\bar{x}, \Sigma)\right)^{T}\left\{e^{\mathcal{L}^{*} \tau} \hat{\rho}(x ; \bar{x}, \Sigma)\right\} d x
$$

There is a connection between particle filters and the moment map. In their basic form particle filters represent the desired evolving probability density function as a sum of several delta functions, or Gaussians, not just as one [6]. However, various simplifications are often used in high-dimensional problems, such as the extended Kalman filter and the ensemble Kalman filter, and these methods have some resemblance to the moment map in spirit.

3.2. The ODE case. The moment map can be defined for deterministic problems of the form

$$
\begin{aligned}
& \frac{d x}{d t}=f(x, y), \\
& \frac{d y}{d t}=g(x, y) .
\end{aligned}
$$

Here $x \in \mathbb{R}^{d}$ and $y \in \mathbb{R}^{m}$, and the randomness is assumed to come entirely from the initial data. In systems characterized by a separation of time scales, it is sometimes the case that one can write an effective reduced model in terms of a subset of (typically slow) variables. Under such appropriate conditions, we might, for example, be interested in finding a map in terms of the first $k$ moments of $x$ alone. Thus the measure $\mu$ must be chosen on $\mathbb{R}^{d} \times \mathbb{R}^{m}$ so that it is uniquely characterized by $X$, the first $k$ moments of $x \in \mathbb{R}^{d}$. It is natural to choose $\mu$ to be an invariant measure for the flow, conditioned by knowledge of the first $k$ moments of $x$; if the flow is Hamiltonian, then a Gibbs measure is often used. We denote the density associated with this measure by $\hat{\rho}(x, y ; X)$. This occurs in the heat bath example considered in the introduction, where $x$ represents coordinate and momentum of the distinguished particle, while $y$ represents the heat bath coordinates and momenta.

Rather than the Fokker-Planck equation (3.3) we have the Liouville equation for propagation of probability densities. This is

$$
\begin{aligned}
\frac{\partial \rho}{\partial t}=-\nabla_{x} \cdot(f \rho)-\nabla_{y} \cdot(g \rho) & :=\mathcal{L}^{*} \rho, \\
\rho(x, y, 0) & =\hat{\rho}(x, y ; X),
\end{aligned}
$$


and we denote the solution by

$$
\rho(x, y, t)=e^{\mathcal{L}^{*} t} \hat{\rho}(x, y ; X) .
$$

In the case of a Dirac mass we take $\hat{\rho}(x, y ; X)=\delta(x-X) \hat{\rho}(y ; X)$, and then the map

$$
\Phi(X)=\int_{\mathbb{R}^{d} \times \mathbb{R}^{m}} x\left\{e^{\mathcal{L}^{*} \tau} \delta(x-X) \hat{\rho}(y ; X)\right\} d x d y .
$$

Here $\hat{\rho}(y ; X)$ is chosen so that $\delta(x-X) \hat{\rho}(y ; X)$ is the density of $\mu$ conditional on $x=X$. Generalization to Gaussian, and higher moment problems, is also possible.

\subsection{General remarks.}

- Notation. We use $X$ to represent a point in the moment space up to some order $k$, which will be made explicit for each particular example we consider. In practice, the coordinates used to describe the moment space are dictated by the particular problem. For example, in our case we use the mean and standard deviation as coordinates when considering $k=2$ and $d=1$. For $k=2$ and $d=2$ we use the two means, the two standard deviations, and the cross correlation as coordinates.

- Usage. In discussing moment maps we often do not distinguish between a point $X$ in moment space and the uniquely determined density $\hat{\rho}(\cdot ; X)$ based on this point. That is, we sometimes speak of the moment map as mapping $X^{n}$ to $X^{n+1}$ and sometimes speak of the moment map as mapping density $\hat{\rho}\left(\cdot ; X^{n}\right)$ to density $\hat{\rho}\left(\cdot ; X^{n+1}\right)$.

- Relation to optimal prediction. The map $\Phi$ can be used to generate an approximate vector field by defining

$$
F(X)=\frac{\Phi(X)-X}{\tau}
$$

For the ODE case (3.6) and $\mu$ a Dirac at $X$, the limit $\tau \rightarrow 0$ coincides with the vector field found by the method of optimal prediction [3]; this is demonstrated in [16]. In general the method of optimal prediction leads to errors which grow linearly in time $T$ [19]. The approach we study here attempts to overcome this error growth by closing the system with a larger number of moments.

- Previous work. For simple problems in chemical kinetics, which are modeled by birthdeath processes, the equation for the first moment is a closed ODE, in the limit of a large number of independent particles, and the moment map studied here then works well in the Dirac mass case [29, 30, 31]. For more complex problems, such as lattice Boltzmann, a closed effective PDE may sometimes be found, using first and second moments, and again, the moment map works well in this case $[40,12,41,9]$. In this paper we study examples where no closure is proved to exist, and demonstrate the properties of the moment map. In particular we study the relevance of fixed points of this map to the identification of metastable states. Although no rigorous analysis is presented, the numerical studies show that the moment map has some merit as an approach for elucidating long-term dynamics of large systems, through low-dimensional dynamical systems studies. 
- Lifting. Initialization of the detailed simulation consistently with coarse-grained observables is the lifting step in equation-free computation [40, 12, 25, 38]. This step is obviously not unique, as there exist many ways of initializing a distribution conditioned on a few of its lower-order moments. Our choice of a particular measure depending only on the lower-order moments allows for a systematic initialization of the fine-scale dynamics, a concept that goes back to Ehrenfest (see $[8,17]$ ), and is an important component of our computational approach. In the case of systems with metastability, different effective dynamics will be deduced (different closures will be obtained) depending (a) on the time scale of the observation (the time horizon of the simulation $\tau$ with the fine scale solver) and (b) on the nature of the lifting from the moments (the choice of $\rho$ ). Over very short times, and initializing with a Dirac delta function, the simulation will effectively sample the local gradient of the well; over medium times, and initializing within one well, one will observe equilibration within this well; and over very long times (no matter what the initialization) one will observe the approach to the equilibrium density. If we want to study the system over medium time scales, it is obviously important to use a time $\tau$ in the construction of the moment map that is short compared to the escape time between wells, but long enough for the noise (dynamics) to allow the sampling of the features of the well bottom. On a longer time scale it is necessary to incorporate the transition time between wells, as is done, for example, in the method of conditional averaging [36].

- Computational savings. The moment map can lead to computational savings in two primary ways:

(i) the map $\Phi$ can be used in finite-dimensional bifurcation and continuation studies;

(ii) the estimated vector field $F$ can be used to advance the moments over several multiples of the time-step $\tau$.

In case (i), savings can arise from using accelerated methods, such as Newton iteration in a continuation environment, to find fixed points. In case (ii), savings arise by considering maps of the type

$$
X^{n+1}=X^{n}+l\left\{\Phi\left(X^{n}\right)-X^{n}\right\}
$$

to advance the moments through $l \tau$ time units, using solution of the full problem (3.1) or (3.6) only over $\tau$ time units. (If $l=1$, this simply reduces to the moment map.) The above formula constitutes a projective forward Euler explicit coarse integrator. Much more sophisticated integrators, including multistep and implicit ones, can also be used; a rigorous analysis of savings in case (ii) has only recently been initiated [13, 14, 35]. These ideas have been applied to a wide range of problems, both deterministic and stochastic; see, e.g., [15, 12, 29, 30, 31, 41, 38, 9, 18, 27, 22, 37, 4] and other references in $[25,26]$. An approach related to case (ii), which can be fully optimized for variance reduction and so forth when explicit time scale separation occurs between the $x$ and $y$ dynamics in (3.8), is outlined in [42]; for a rigorous analysis see [7].

4. Examples. Here we explore a series of examples of moment maps. We start with examples based on the Ornstein-Uhlenbeck (OU) process, for which explicit representation is possible. In these cases the moment maps are linear. We then proceed to the more interesting 

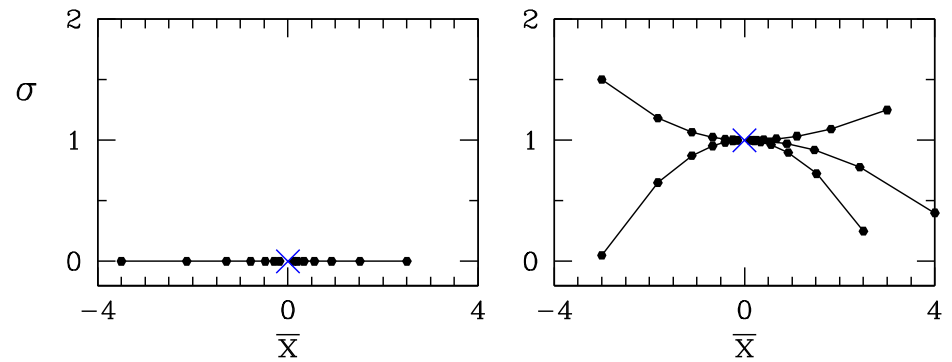

Figure 6. Phase portraits for first-order (left) and second-order (right) moment maps for the OU process. Trajectories for these linear maps are shown starting from several different initial conditions. Parameters are $\alpha=1, \lambda=2$, and $\tau=0.5$. See also the accompanying animations (63866_01.gif [714KB], 63866_02.gif [1.4MB], and 63866_03.gif [2.5MB]).

nonlinear maps arising from systems with double-well potentials.

4.1. The OU process. Consider the OU process

$$
\frac{d x}{d t}=-\alpha x+\sqrt{\lambda} \frac{d W}{d t} .
$$

This is essentially the simplest example of (3.1) and corresponds to (2.5) in the case of a quadratic potential.

The exact solution of this process is

$$
x(t)=e^{-\alpha t} x(0)+\sqrt{\lambda} \int_{0}^{t} e^{-\alpha(t-s)} d W(s) .
$$

For a first-order moment map on $\bar{x}$, we take initial data with Dirac measure with density $\hat{\rho}(x ; \bar{x})=\delta(x-\bar{x})$. The map on the first moment $\bar{x}$ is explicitly

$$
\Phi(\bar{x})=\mathbb{E} x(\tau)=\int x\left\{e^{\mathcal{L}^{*} \tau} \delta(x-\bar{x})\right\} d x .
$$

From (4.2) we have

$$
\Phi(\bar{x})=e^{-\alpha \tau} \bar{x} .
$$

The map $\Phi$ is linear and has a unique globally attracting fixed point at $\bar{x}=0$. Figure 6 shows a phase portrait for this simple map.

Before discussing this we consider the second-order moment map with mean $\bar{x}$ and standard deviation $\sigma$ as coordinates. In this case the initial data has density

$$
\hat{\rho}(x ; \bar{x}, \sigma)=\frac{1}{\sqrt{2 \pi} \sigma} e^{-\frac{(x-\bar{x})^{2}}{2 \sigma^{2}}} .
$$

From (4.2) we then have that the moment map on $(\bar{x}, \sigma)$ is given by

$$
\begin{aligned}
& \Phi_{1}(\bar{x}, \sigma)=e^{-\alpha \tau} \bar{x}, \\
& \Phi_{2}(\bar{x}, \sigma)=\left\{e^{-2 \alpha \tau} \sigma^{2}+\frac{\lambda}{2 \alpha}\left[1-e^{-2 \alpha \tau}\right]\right\}^{\frac{1}{2}} .
\end{aligned}
$$


While not a linear map on $(\bar{x}, \sigma)$, this is linear on $\left(\bar{x}, \sigma^{2}\right)$. Figure 6 shows a phase portrait for map (4.4). The map has the unique fixed point $\left.(\bar{x}, \sigma)=\left(0, \sqrt{\{} \frac{\lambda}{2 \alpha}\right\}\right)$, which is globally attracting.

The solution to (4.2) is Gaussian if $x_{0}$ is Gaussian. Hence the second-order moment map (4.4) gives exact time $\tau$ samples of the distribution of the SDE. This unique fixed point of the map characterizes the unique invariant (Gaussian) measure of (4.1). In contrast, the first-order moment map, with $\mu$ a Dirac, can only approximate the solution. Although this map does not quantitatively represent the solution, since it contains no information about the width of the measure, it captures the correct dynamics of the first-order moment and shows that probability mass initially far from the origin will be transported towards the origin.

4.2. One-dimensional SDE: The double-well potential. We now present a detailed study of moment maps for the one-dimensional SDE (2.5) with double-well potential. In this section we focus on the dynamics of these maps using numerical simulations. In the next section we analyze the maps, in particular the nonlinearity of the maps, in more detail.

After suitable scaling, SDE (2.5) can be rewritten in the notation of (3.1) as

$$
\dot{x}=-V^{\prime}(x)+\dot{W} .
$$

We consider the double-well potential

$$
V^{\prime}(x)=x\left(x^{2}-\mu\right)+\nu .
$$

For $\nu=0$ the potential is symmetric about zero, and for $\nu$ small this symmetry is weakly broken. The potential has two local minima for $|\nu|<2(\mu / 3)^{3 / 2}$ and one minimum otherwise.

The first-order (Dirac-based measure) and second-order (Gaussian-based measure) moment maps for this equation are nonlinear. In particular, these maps have multiple stable fixed points that undergo bifurcations as the potential ( $\mu$ or $\nu$ ) is varied. We are interested in these fixed points and their stable and unstable manifolds as a function of $\mu$ for $\nu$ fixed. We shall consider two cases, the slightly asymmetric case $\nu=0.3$ and the symmetric case $\nu=0$. We resort solely to numerical studies of the moment maps throughout this section. In brief, we use Monte Carlo simulations to evolve densities forward over time interval $\tau$, as in the evolution from $\hat{\rho}$ to $\rho$ in Figure 5. This numerically determines the moment maps. By employing additional techniques, we can compute steady states and bifurcations. The effective fixed point, bifurcation, and continuation calculations require estimates of the Jacobian of the moment map (or its action). This is achieved by using nearby initializations of the moment map (see [29, 30] as well as [40, 25, 23] and the monograph [24] for matrix-free implementations of Newton-GMRES).

We first consider the moment maps for the slightly asymmetric potential with representative values of $\mu$ and map time $\tau$. Figure 7 shows phase portraits for both first-order and second-order moment maps. Each map has three fixed points. These are shown together with unstable, and for the second-order map also stable, manifolds of the unstable fixed point.

Figure 8 shows one iteration of both the first- and second-order maps. Consider the firstorder map. The initial density $\rho(0)=\hat{\rho}(x ; \bar{x})$ is a Dirac delta at $\bar{x}$, here a point slightly to the right of the unstable fixed point near zero. After time $\tau=0.1$ the mean of the density has 

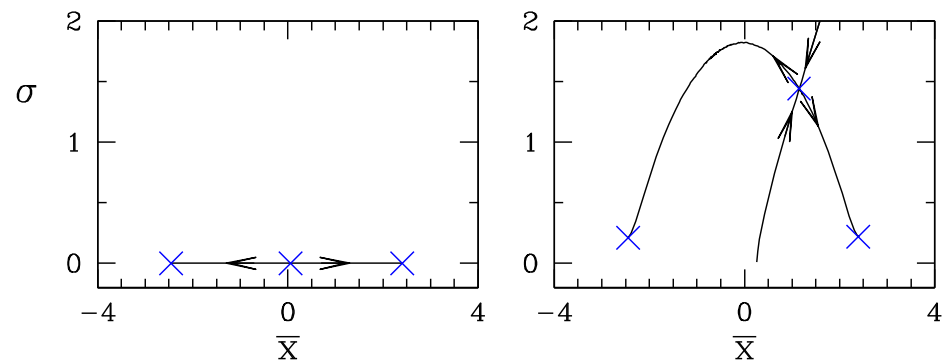

Figure 7. Phase portraits for first-order (left) and second-order (right) moment maps for the one-variable SDE in the case of a slightly asymmetric potential. Fixed points are indicated by crosses. The stable (for second-order map) and unstable manifolds of the middle fixed point are shown. Note, the stable manifold of the saddle in the second-order map does not pass through the middle fixed point of the first-order map. Parameters are $\mu=6, \nu=0.3, \tau=0.1$.
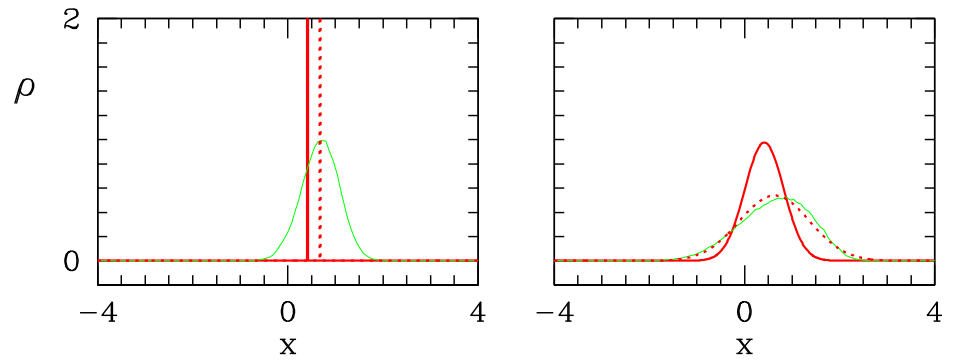

Figure 8. One iteration of the first and second order maps whose phase portraits are shown in Figure 7. The bold red curve shows $\rho(0)=\hat{\rho}\left(x ; X^{n}\right)$, the thin green curve shows $\rho(\tau)$, and the dash bold red curve shows $\hat{\rho}\left(x ; X^{n+1}\right)$. (See Figure 5.)

moved to the right, and hence the map takes $\bar{x}$ to the right in this case. The density spreads considerably, but over $\tau=0.1$ it remains nearly symmetric (the mean is indistinguishable from the maximum). The initial density for the next iteration is a Dirac delta displaced to the right.

For the second-order map the initial density $\rho(0)=\hat{\rho}(x ; \bar{x})$ is a Gaussian centered at $\bar{x}$ with width $\sigma$. Here $(\sigma, \bar{x})=(0.418,0.409)$ corresponds to a point on the lower branch of the stable manifold of the saddle fixed point. After $\tau=0.1$ time units the density has spread, and the mean has moved slightly to the right. The map thus corresponds to substantial increase in $\sigma$ and small increase in $\bar{x}$. The density $\rho(\tau)$ is slightly non-Gaussian, as can be seen in comparison with the initial (Gaussian) density for the next iteration.

Figure 9 shows all fixed points in Figure 7 . In each case we plot the density $\hat{\rho}(\cdot ; X)$ corresponding to each fixed point in moment space as well as the density $\rho(\tau)$. The stable fixed points of the second-order map are the metastable measures centered in each well. Specifically, the Gaussian measures $\hat{\rho}(x ; \bar{x}, \sigma)$ corresponding to the stable fixed points are indistinguishable from the evolved densities $\rho(\tau)$. As we shall show this is independent of $\tau$ over a very large range of $\tau$. Intuitively this is because on any time scale, up to the very long time scale needed 

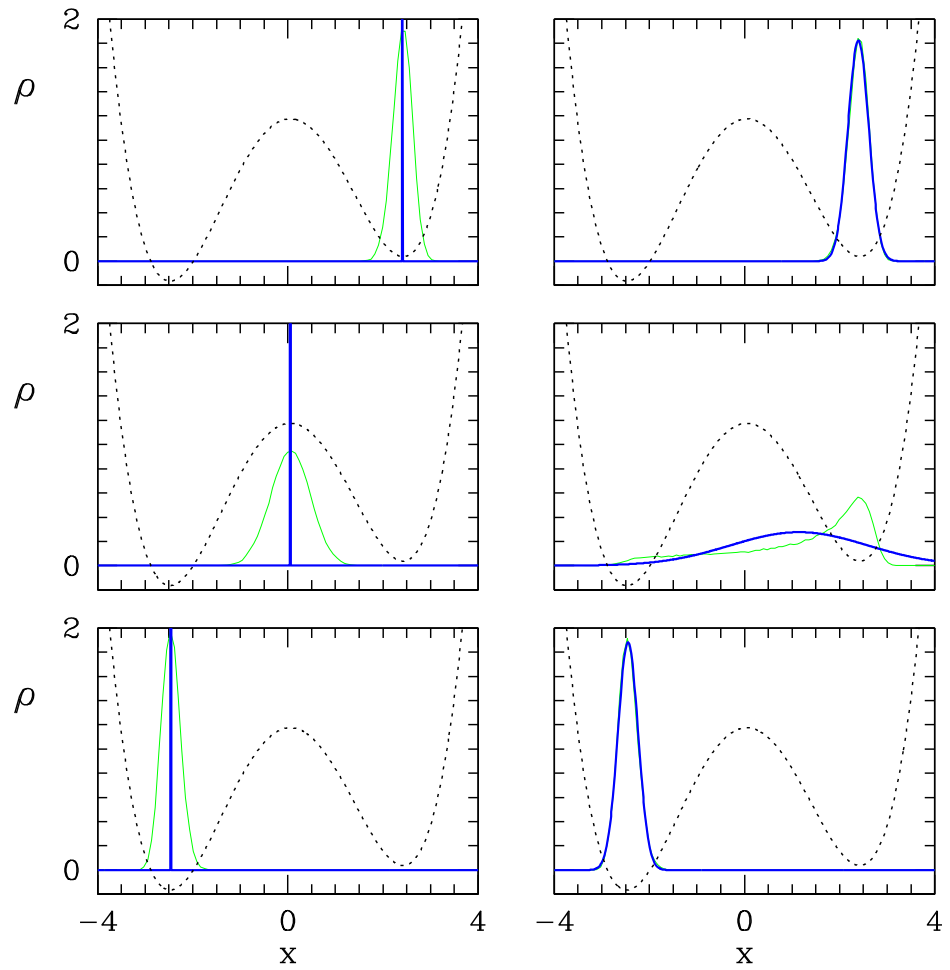

Figure 9. Densities $\hat{\rho}(\cdot ; X)$, shown with bold blue curves, corresponding to fixed points for the first-order (left) and second-order (right) moment maps shown in Figure 7. Thin green curves show the evolved density $\rho(\tau)$ for each case. (For the two stable fixed points of the second-order moment map the evolved density $\rho(\tau)$ is indistinguishable from $\hat{\rho}(\cdot ; X)$.) The potential $V(x)$ is shown as a dashed curve.

to reach the equilibrium distribution, these Gaussian measures are approximately invariant. The stable fixed points of the first-order map are not invariant measures. However, starting from initial condition $\hat{\rho}(x ; \bar{x})=\delta(\bar{x})$, where $\bar{x}$ is indistinguishable from the potential minimum, the density simply fills out (symmetrically), the (locally quadratic) well bottom. Note that $\tau=0.1$ is close to, but not quite, the time necessary to reach the metastable density starting from the Dirac measure.

The unstable fixed points for the maps are understood as follows. For the first-order map, the unstable fixed point is at the local maximum of the potential. Starting from a Dirac delta $\hat{\rho}(x ; \bar{x})=\delta(\bar{x})$, the density spreads symmetrically, since the maximum is locally quadratic and hence symmetric. Hence after time $\tau=0.1$ the mean is still at local maximum of the potential. Only for times $\tau$ long enough for the density to fill the two wells, and hence have a mean different from the local maximum, would the fixed point be different from the local maximum. For such times the fixed point would approximately be the mean of the equilibrium density. For the second-order map, the saddle fixed point corresponds to a Gaussian $\hat{\rho}(x ; \bar{x}, \sigma)$, which is quite broad. The evolved density $\rho(\tau)$ is far from Gaussian; it simply has the property that its first two moment agree with those of the initial Gaussian. The saddle fixed point is quantitatively sensitive to the map time $\tau$ (see below). Qualitatively, however, for any value 

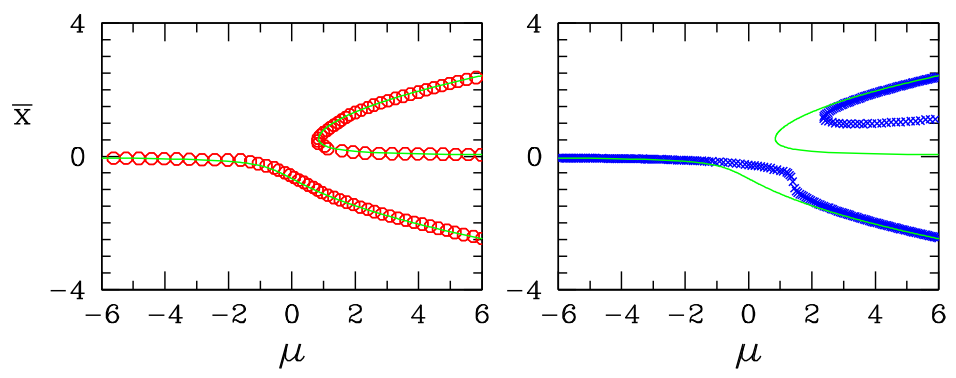

Figure 10. Bifurcation diagram for first-order (left) and second-order (right) moment maps for the onevariable SDE in the case of a slightly asymmetric potential. Lines show local extrema of the potential. Parameters are $\nu=0.3$ and $\tau=0.1$.

of $\tau$ the saddle fixed points are broad Gaussians.

The stable fixed points of both maps correspond to metastable states (measures) of the underlying process. The metastable states are very nearly Gaussian measures, because the wells are locally quadratic, and they are thus well captured by the low-dimensional moment maps: the densities corresponding to the stable fixed points of the second-order map are virtually indistinguishable from the metastable states. The fixed points of the first-order map capture the means of the metastable distributions. The stable fixed points are insensitive to the value of $\tau$ over a large range of $\tau$. (See Figure 12 below.) This lack of sensitivity to $\tau$ suggests that these fixed points are meaningful characteristics of the observed dynamics over a range of observation time scales. The unstable (saddle) fixed point for the first-order moment map is also insensitive to the value of $\tau$, but the unstable fixed point for the second-order map is sensitive to $\tau$. This suggests that the fixed points of the first-order moment map provide a useful description of the dynamics (for relatively short times) close to both the saddle and the well bottoms; the second-order map fixed points provide a meaningful description of the effective dynamics close to the bottoms of the two wells, but not in the neighborhood of the saddle; this is essentially because a reduced equation in terms of the second-order moments does not appear to successfully close in the neighborhood of the saddle.

We now turn to the behavior of the fixed points as a function of well depth. Figure 10 shows bifurcation diagrams for each moment map as functions of $\mu$, including $\mu$ for which the potential has a single well (basically $\mu<0$ ). Local extrema of the potential are shown for comparison. The right-most end of each bifurcation diagram $(\mu=6)$ corresponds to the phase portraits just considered. The fixed points for the first-order map follow the extrema of the potential closely for all $\mu$, including near the saddle-node bifurcation. The second-order fixed points do not. In Figure 11 we show fixed points in cases where the potential is far from locally quadratic. One case is $\mu=0$, and the other is $\mu=2.4$, very near the value corresponding to the saddle-node bifurcation of the second-order map. In both cases the first-order fixed point is at the potential minimum, while the mean of the second-order fixed point is noticeably different from the minimum, as can also be seen in Figure 10.

We show in Figure 12 how steady states for the second-order moment map are affected by the choice of the map time $\tau$. To understand what the figure shows, it is helpful to first consider the fixed points for $\mu<0$. Neither $\bar{x}$ nor $\sigma$ varies significantly with $\tau$, and $\sigma$ is 

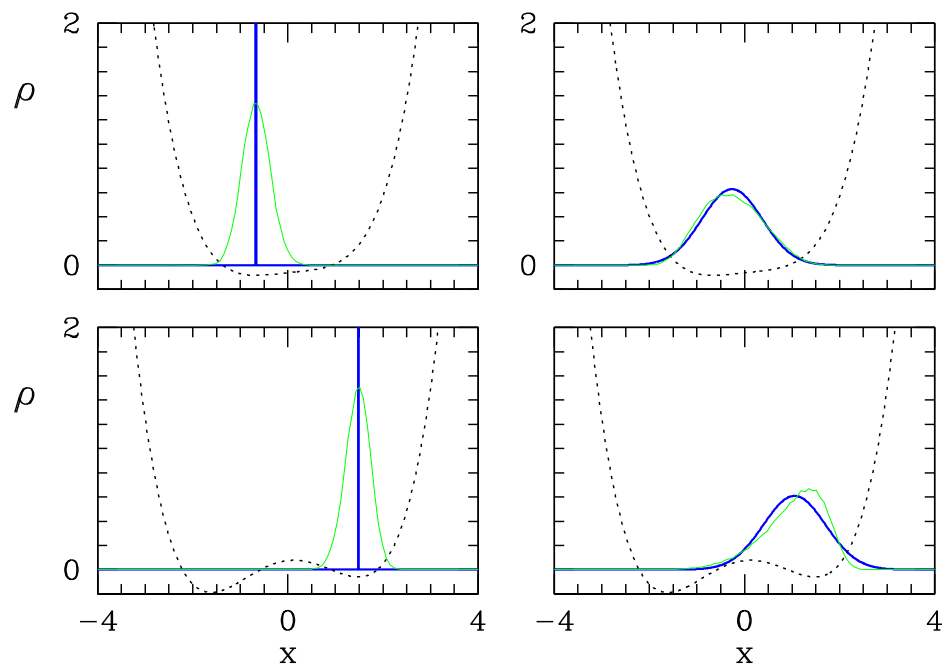

Figure 11. Densities, shown with bold blue curves, corresponding to fixed points for first-order (left) and second-order (right) moment maps at two values of $\mu$ where the nonquadratic aspect of the potential is apparent. Thin green curves show the evolved density $\rho(\tau)$ for each case. The top case is $\mu=0$. The bottom is $\mu=2.4$, very near the saddle-node bifurcation for the second-order map in Figure 10. The potential $V(x)$ is shown as a dashed curve.

not large. While not as easy to see, the stable fixed points for $\mu \gtrsim 2$ behave similarly. The stable fixed points correspond to the uppermost and lowermost branches in the $\bar{x}$-plot and the bottom branch in the $\sigma$-plot. Both stable fixed points have approximately the same, relatively small, value of $\sigma$. The intermediate, generally unstable, fixed points have larger values of $\sigma$; these vary quantitatively and qualitatively with $\tau$. The number of unstable fixed points can change with $\tau$; e.g., at $\mu=6, \tau=0.1$ there is one saddle fixed point between the two stable fixed points (Figure 7), whereas at $\mu=6, \tau=1.0$ there are three fixed points between the two stable points (similar to Figure 14 below). The conclusion is that the stable fixed points of the second-order moment map which correspond to stable or metastable measures of the SDE are largely insensitive to the time $\tau$ used to define the map. The other fixed points typically correspond to broad distributions and depend quantitatively on $\tau$. This dependence of the fixed points of the moment map on $\tau$ suggests that they are not useful features of the effective dynamics - which in turn suggests that the effective behavior does not usefully close at the second moment level in the neighborhood of these fixed points.

A further way to quantify the effective closure at a given level is to examine how closely two successive iterates of the moment map agree with one iterate using twice the map time. Specifically, one can consider whether $\Phi_{\tau}\left(\Phi_{\tau}(X)\right) \simeq \Phi_{2 \tau}(X)$ for some particular point $X$ in moment space and some chosen map time $\tau$. If the two are nearly equal, then the system is effectively closed with the given number of moments in the vicinity of point $X$. We illustrate this in Figure 13 for the second-order moment map. We have computed $\| \Phi_{\tau}\left(\Phi_{\tau}(\bar{x}, \sigma)\right)-$ $\Phi_{2 \tau}(\bar{x}, \sigma) \|_{2}$ for a large number of points in moment space with $\tau=0.1$. The norm of the difference is shown in greyscale. Everywhere in the white region the norm is very small. Starting from any point in the white region, two map iterations over time $\tau$ and one iteration 

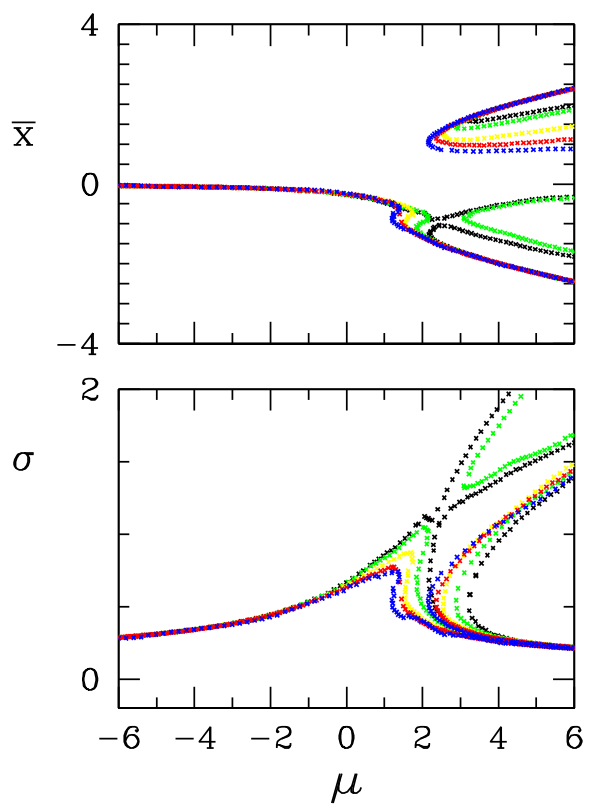

Figure 12. Bifurcation diagram for a second-order moment map for the one-variable SDE for a variety of values of $\tau: \tau=0.05$ blue, $\tau=0.1$ red, $\tau=0.2$ yellow, $\tau=0.5$ green, $\tau=1.0$ black. Other parameters are $\mu=6$ and $\nu=0.3$.

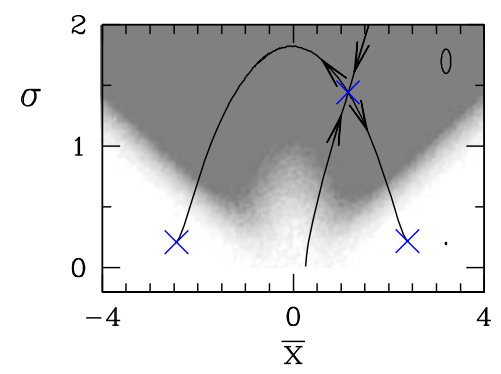

Figure 13. Effective closure for second-order moment maps for the one-variable SDE in the case of a slightly asymmetric potential. Shown in greyscale is $\left\|\Phi_{\tau}\left(\Phi_{\tau}(\bar{x}, \sigma)\right)-\Phi_{2 \tau}(\bar{x}, \sigma)\right\|_{2}$ as a function of $\bar{x}, \sigma$, for $\tau=0.1$. White corresponds to a norm less than 0.01, while dark grey corresponds to a norm greater than 0.1 . For reference, two ellipses are plotted $(a t \bar{x}=3.2)$ : a very small one in the white region corresponds to a neighborhood of size 0.01, and one in the grey region corresponds to a neighborhood of size 0.1. The phase portrait from Figure 7 is also shown. Parameters are $\mu=6, \nu=0.3, \tau=0.1$.

over time $2 \tau$ lie within a neighborhood of size shown. As expected, the white region includes the two stable fixed points. In the grey region the norm is quite large. Here two map iterations over time $\tau$ and one iteration over time $2 \tau$ lie further apart than the neighborhood shown in the grey region. This indicates that is the system does not close in the Gaussian approximation.

Finally we consider the moment maps for the symmetric double-well potential. Figure 14 shows phase portraits, similar to Figure 7 for the asymmetric case, while Figure 15 shows fixed 

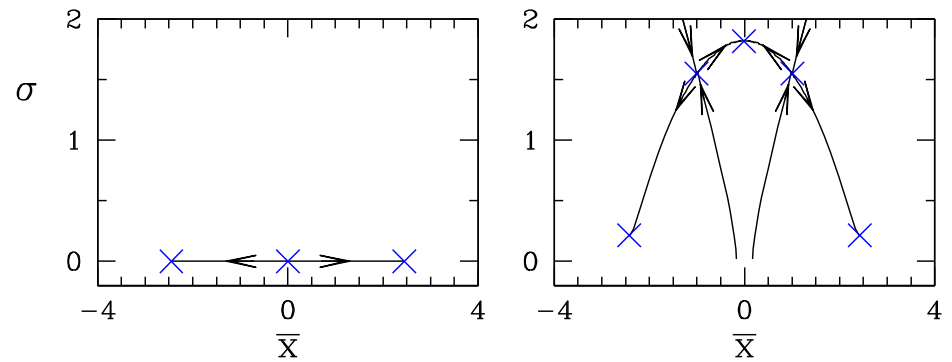

Figure 14. Phase portrait for first-order (left) and second-order (right) moment maps for the one-variable $S D E$ in the case of a symmetric potential. Fixed points are indicated by crosses. The stable (for second-order map) and unstable manifolds of the saddle fixed point are shown. Parameters are $\mu=6, \nu=0, \tau=0.1$.
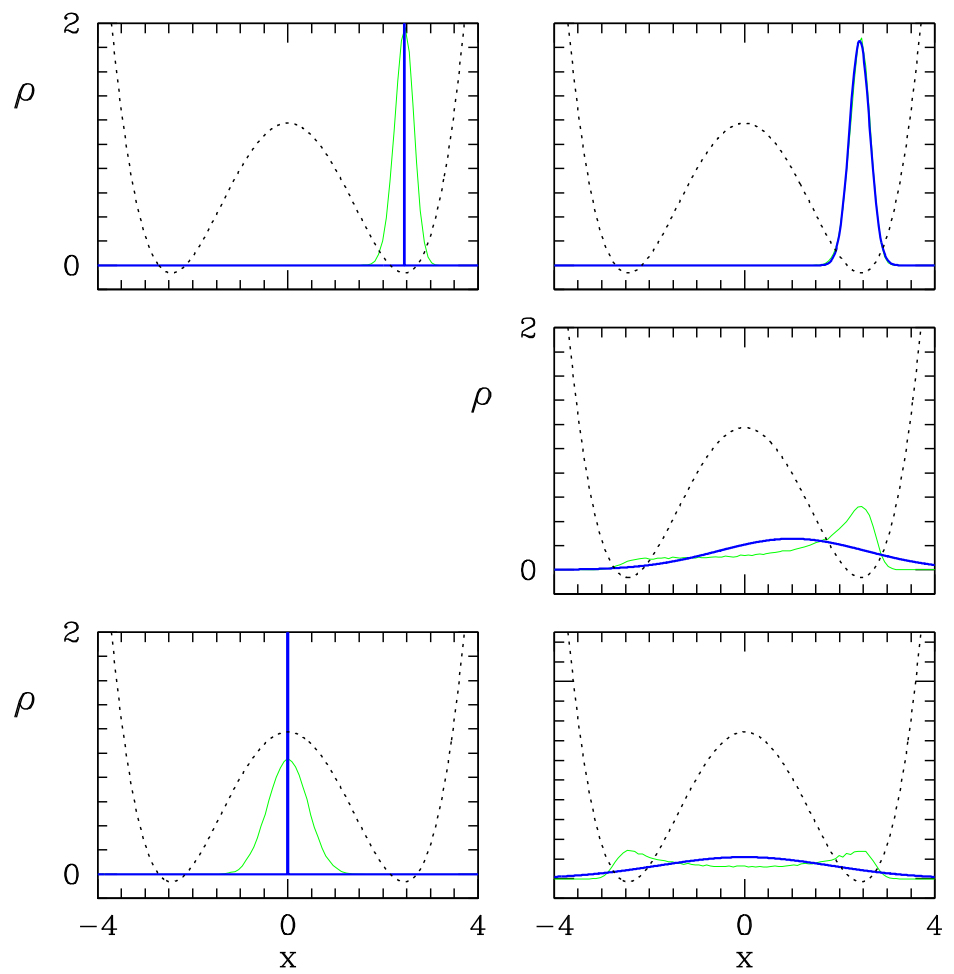

Figure 15. Fixed points for first-order (left) and second-order (right) moment maps shown in Figure 14. Same conventions as in Figure 9. Top plots show the right stable fixed point. The middle plot shows the right saddle for the second-order map. The bottom plot shows the middle fixed point (saddle for the first-order map and stable fixed point for the second-order map). The other points in Figure 14 are obtained by symmetry.

points, similar to Figure 9 for the asymmetric case. Figure 16 shows a bifurcation diagram as a function of $\mu$. An important observation from the data presented in the symmetric case is that the moment map produces stable equilibria that are far from metastable states. The Gaussian measures corresponding to these fixed points are very broad. 

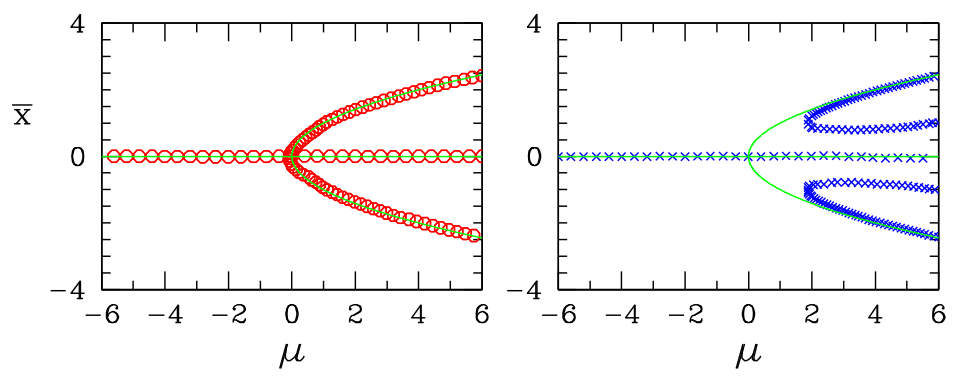

Figure 16. Bifurcation diagram for first-order (left) and second-order (right) moment maps for the case of the symmetric potential. Lines show local extrema of the potential. Parameters are $\nu=0$ and $\tau=0.1$.

4.3. Metastability and the double well potential. We now present some analysis of the nonlinear map. Consider the SDE (4.6). The adjoint of the generator for this process is

$$
\mathcal{L}^{*} \phi(x)=\frac{d}{d x}\left\{V^{\prime}(x) \phi(x)\right\}+\frac{1}{2} \frac{d^{2} \phi}{d x^{2}}(x)
$$

The equation has a unique invariant density $\rho_{\infty}$, in the null-space of $\mathcal{L}^{*}$, given explicitly by

$$
\rho_{\infty}(x)=\mathcal{Z}^{-1} \exp \{-2 V(x)\}, \quad \mathcal{Z}=\int_{\mathbb{R}} \exp \{-2 V(x)\} d x .
$$

The operator $\rho_{\infty}^{-\frac{1}{2}} \mathcal{L}^{*} \rho_{\infty}^{\frac{1}{2}}$ is self-adjoint in the space $L^{2}(\mathbb{R})$ (see Proposition 2.2 of [21]). We let $\langle\cdot, \cdot\rangle$ denote the weighted $L^{2}(\mathbb{R})$ inner product

$$
\langle\theta, \psi\rangle=\int_{\mathbb{R}} \frac{\theta(x) \psi(x)}{\rho_{\infty}(x)} d x
$$

and we write the eigenvalue problem

$$
\mathcal{L}^{*} \phi_{j}(x)=\lambda_{j} \phi_{j}(x)
$$

with eigenvalues ordered so that

$$
0=\lambda_{0} \geq \lambda_{1} \geq \lambda_{2} \geq \cdots .
$$

We may choose the normalization

$$
\left\langle\phi_{j}, \phi_{k}\right\rangle=\delta_{i j}
$$

Since $\phi_{0}(x)=\rho_{\infty}(x)$, we have

$$
\int_{\mathbb{R}} \phi_{0}(x) d x=1, \quad \int_{\mathbb{R}} \phi_{j}(x) d x=0, \quad j \geq 1 .
$$


Now, the solution $\rho(x, t)$ of the Fokker-Planck equation can be expanded in the eigenfunctions $\phi_{j}$ as

$$
\rho(x, \tau)=\sum_{j=0}^{\infty} a_{j}(t) \phi_{j}(x),
$$

where

$$
a_{j}(t)=a_{j}(0) e^{\lambda_{j} t}
$$

Notice that $a_{0}(0)=1$ in all cases, because $\rho(x, 0)$ is a probability density function.

Assume that $V(x)$ in (4.6) is a double-well potential with deep wells relative to the noise. Then the analysis and numerical evidence in $[2,21,32]$ suggest that it is reasonable to assume that

$$
\epsilon:=\lambda_{1} / \lambda_{2} \ll 1
$$

Thus there exists a spectral gap and, noting that $\lambda_{0}=0$, this suggests that after times $\tau$ of order $-1 / \lambda_{2}$ the density $\rho(x, t)$ can be well approximated by only the first two eigenfunctions $\phi_{0}$ and $\phi_{1}$.

Assuming that $V^{\prime \prime} \neq 0$ at the two well bottoms, then $\rho_{\infty}(x)$ is well approximated as the weighted sum of two Gaussians $g_{ \pm}(x)$; this may be verified from (4.8). We may assume that the Gaussians are normalized to be probability densities, and then define their means and standard deviations by

$$
\int_{\mathbb{R}} g_{ \pm}(x) d x=1, \quad \int_{\mathbb{R}} x g_{ \pm}(x) d x=\bar{g}_{ \pm}, \quad \int_{\mathbb{R}}\left[x-\bar{g}_{ \pm}\right]^{2} g_{ \pm}(x) d x=\bar{\sigma}_{ \pm}^{2} .
$$

We then write

$$
\phi_{0}(x)=\rho_{\infty}(x) \approx \alpha g_{+}(x)+(1-\alpha) g_{-}(x),
$$

where $\alpha$ determines the relative weight of the two Gaussian contributions. Furthermore, by orthonormality, it may be shown that

$$
\phi_{1}(x) \approx \sqrt{\{\alpha(1-\alpha)\}\left[g_{+}(x)-g_{-}(x)\right]}
$$

We can now use the approximations (4.10), (4.11) to understand the nonlinearity in the moment map for the case of the double-well potential. We focus on the second-order map. In this case $\rho(x, 0)=\hat{\rho}(x ; \bar{x}, \sigma)$ will be Gaussian. For a sufficiently large spectral gap, (4.9), we can choose a map time $\tau$ long on the scale of $1 / \lambda_{2}$, while short on the scale of $1 / \lambda_{1}$. Specifically, letting $T=-\lambda_{2} \tau$, we have

$$
\rho(x, \tau)=\phi_{0}(x)+a_{1}(0) e^{-\epsilon T} \phi_{1}(x)+O\left(e^{-T}\right) .
$$

Given small $\epsilon \ll 1$, we can choose $T$ sufficiently large that $e^{-T} \ll 1$ while $\epsilon T \ll 1$. Then letting

$$
\beta=a_{1}(0) \sqrt{\{\alpha(1-\alpha)\}}
$$



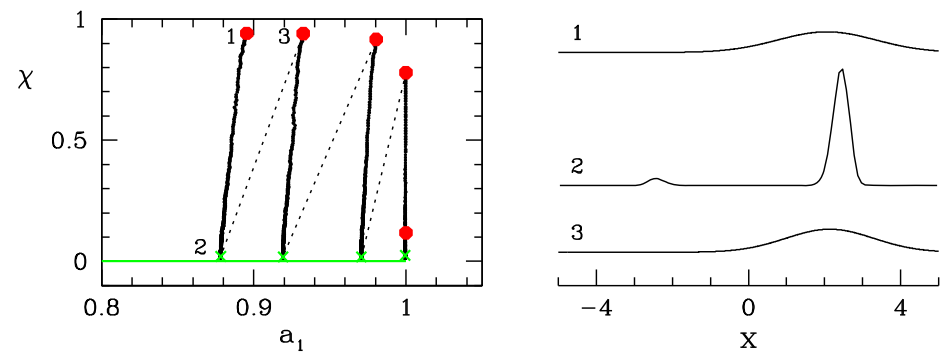

Figure 17. Iterates of the moment map as seen in $a_{1}, \chi$ coordinates. Red dots show points with Gaussian densities $\hat{\rho}(x ; \bar{x}, \sigma)$. The Gaussian densities corresponding to points labeled 1 and 3 are shown to the right. The evolution of the density over time $\tau$ is shown by bold curves (actually fine series of bold points) with green crosses indicating the final time. The density corresponding to point 2 is shown to the right. Dashed lines indicate the projections back to Gaussian density, which preserve the mean and standard deviation of the distribution. Parameters are $\mu=6, \nu=0$, and $\tau=1.0$.

we have

$$
\begin{aligned}
\rho(x, \tau) & \approx(\alpha+\beta) g_{+}(x)+(1-\alpha-\beta) g_{-}(x) \\
& =\gamma g_{+}(x)+(1-\gamma) g_{-}(x),
\end{aligned}
$$

where $\gamma=\alpha+\beta$.

The key to the dynamics of the moment map is manifest in the formula (4.12). Given any Gaussian with mean and standard deviation $\left(\bar{x}_{0}, \sigma_{0}\right)$, we project the density $\rho(x, 0)=$ $\hat{\rho}\left(x ; \bar{x}_{0}, \sigma_{0}\right)$ onto the basis $\left\{\phi_{j}(x)\right\}_{j=1}^{\infty}$. Then the evolution over appropriate time $\tau$ results in $\rho(x, \tau)$ containing only $\phi_{0}$ and $\phi_{1}$ and is given by (4.12), so that

$$
\begin{aligned}
\bar{x}_{1} & =\gamma \bar{g}_{+}+(1-\gamma) \bar{g}_{-}, \\
\sigma_{1}^{2} & =\beta \bar{\sigma}_{+}^{2}+(1-\beta) \bar{\sigma}_{-}^{2}+(1-\beta) \beta\left(\bar{g}_{+}-\bar{g}_{-}\right)^{2} .
\end{aligned}
$$

Since $\beta, \gamma$ depend nonlinearly on $\left(\bar{x}_{0}, \sigma_{0}\right)$, through $\alpha$ and $a_{1}(0)$, we have a nonlinear map $\left(\bar{x}_{0}, \sigma_{0}\right) \rightarrow\left(\bar{x}_{1}, \sigma_{1}\right)$. In principle this map can be computed explicitly, though this requires knowing the eigenfunctions $\phi_{1}$ and $\phi_{0}$ accurately.

This analysis can be applied to our computational studies of the double-well potential to gain further insight into the moment map. We employ the symmetric double-well potential considered at the end of section 4.2. In this case the eigenfunctions $\phi_{1}$ and $\phi_{0}$ are accurately approximated by (4.10) and (4.11) with $\alpha=1 / 2$. We use $\tau=1$ in the computations that follow. (We shall see that $\lambda_{2} \simeq-5$, so that $\tau \lambda_{2} \simeq-5$.)

Figure 17 shows the moment map in the coordinates suggested by the preceding analysis. The corresponding evolution of the density is shown for one iterate of the map. We wish to view the evolution of the density $\rho(x, t)$ in terms of the amplitudes $a_{j}(t)$ of projections onto the eigenfunctions $\phi_{j}$. We know that $a_{0}(t) \equiv 1$, so there is no need to show this amplitude. The essential amplitude is $a_{1}$. The effect of all the higher amplitudes $a_{j}, j>1$, can be summarized 


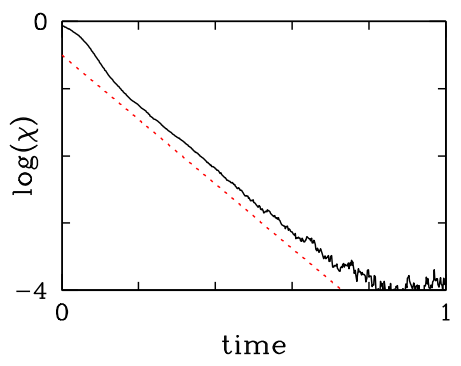

Figure 18. Exponential decay of higher modes. Dashed curve has slope -4.8 .

by a single scalar $\chi$ defined as

$$
\chi \equiv\left\|\sum_{j=2}^{\infty} a_{j} \phi_{j}\right\|_{2}=\left\|\rho-a_{0} \phi_{0}-a_{1} \phi_{1}\right\|_{2},
$$

with $\rho=\rho(\cdot, \tau)$.

Consider point 1 in Figure 17. To the right is shown the Gaussian density $\rho(0)=$ $\hat{\rho}\left(x ; \bar{x}_{0}, \sigma_{0}\right)$ determined by a point $\left(\bar{x}_{0}, \sigma_{0}\right)$ in moment space. This density has a significant projection onto the higher modes $\phi_{j}, j>1$, and $\chi$ is significantly greater than zero. From the analysis we expect the density to evolve such that $\chi$ decays to zero on a time scale faster than the dynamics of $a_{1}$. The thick black curves in Figure 17 show this evolution. There is little change in $a_{1}$ as the system evolves toward $\chi=0$. The decay to $\chi=0$ is ultimately exponential, as is shown in Figure 18. From this we estimate that $\lambda_{2} \simeq-5$ and thus $\exp \left(\lambda_{2} \tau\right) \simeq 0.007$.

After time $\tau=1$ the system is at point 2 with corresponding density shown at the right. The resulting density is no longer Gaussian as expected from (4.12). The secondorder moment map is obtained by determining $\left(\bar{x}_{1}, \sigma_{1}\right)$, the mean and standard deviation of density 2. For the next iteration of the map one constructs a Gaussian density with this mean and standard deviation (point 3 in Figure 17) and repeats. The dashed line connecting points 2 and 3 illustrates this. Thus the moment map takes Gaussian $\hat{\rho}\left(x ; \bar{x}_{0}, \sigma_{0}\right)$ determined by $\left(\bar{x}_{0}, \sigma_{0}\right)$ (point 1$)$ to $\hat{\rho}\left(x ; \bar{x}_{1}, \sigma_{1}\right)$ determined by $\left(\bar{x}_{1}, \sigma_{1}\right)$ (point 3 ) where the map can be again be iterated. Note that the evolution from point 1 to point 2 is due to linear flow of the Fokker-Planck equation. Nonlinearity results from the projection of density at point 2 back to $\left(\bar{x}_{1}, \sigma_{1}\right)$; i.e., going from point 2 to point 3 introduces nonlinearity into the map. Further note that the mean has moved slightly to the right after one iteration of the map. Hence, on the next iteration less of the mass will move into the left well (as in point 2). In this way the map stabilizes the metastable states corresponding to localization of density into a single well.

Now consider exactly the same evolution seen in the moment-map coordinates $(\bar{x}, \sigma)$ in Figure 19. The green curve is that generated by $a_{0}=1,-1 \leq a_{1} \leq 1, a_{j}=0, j>1$. This curve is also shown as green in Figure 17. This is the slow manifold for the system. Starting from point $\left(\bar{x}_{0}, \sigma_{0}\right)$, point 1 , the evolution of $(\bar{x}, \sigma)$ as $\rho(x, t)$ evolves is shown in bold. This is the decay of the modes $\phi_{j}, j>1$. After time $\tau$ the system reaches $\left(\bar{x}_{1}, \sigma_{1}\right)$, point 2 . Even 


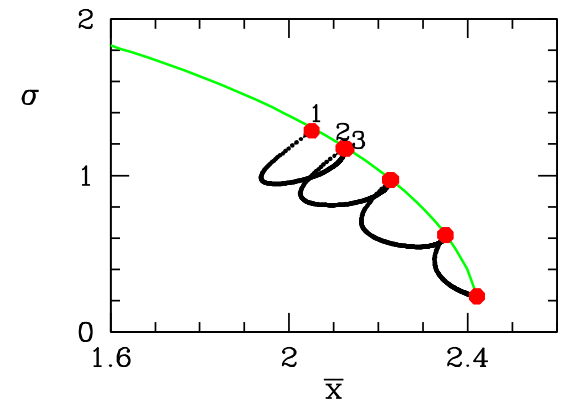

Figure 19. Iterates of the moment map seen in $(\bar{x}, \sigma)$ coordinates. The points correspond to exactly the same points as in Figure 17. The bold curves (actually a fine series of bold points) show the evolution of $(\bar{x}, \sigma)$ as the density evolves between iterates of the map. The green curve is the slow manifold $(\chi=0)$, also shown in Figure 17.

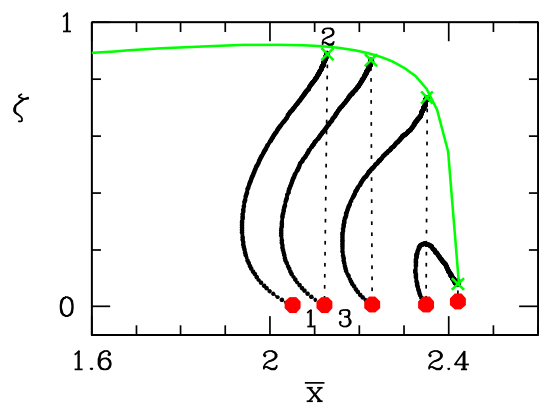

Figure 20. Iterates of the moment map seen in $(\bar{x}, \zeta)$ coordinates. The points correspond to exactly the same points as in Figures 17 and 19. The bold curves (actually a fine series of bold points) show the evolution of $(\bar{x}, \zeta)$ as the density evolves between iterates of the map. The green curve is the slow manifold.

though the transient dynamics is such as to move initially away from the slow manifold, the evolution brings the system back, as it must. The projection back to Gaussian density does not change $\bar{x}$ and $\sigma$, so points 2 and 3 appear the same in Figure 19.

We know that as the system evolves from point 1 to point 2 the density became nonGaussian. We show this in Figure 20 by showing the dynamics out of the $(\bar{x}, \sigma)$-plane. Here $\zeta$ is given by

$$
\zeta \equiv\|\rho-\hat{\rho}(\cdot, \bar{x}, \sigma)\|_{2},
$$

with $\rho=\rho(\cdot, \tau)$, and is similar in spirit to $\chi \cdot \zeta$ is a measure of how far the density is from Gaussian, whereas $\chi$ is a measure of how far the density is from the slow manifold. However, these have opposite roles and behavior. Gaussian densities correspond to $\zeta=0$, but since such densities are not in general on the slow manifold they correspond to $\chi \neq 0$.

Starting from point 1 in Figure 20, $\zeta$ is necessarily zero since the density (Figure 17 point 1 ) is Gaussian. As the system evolves toward the slow manifold and becomes non-Gaussian, $\zeta$ 


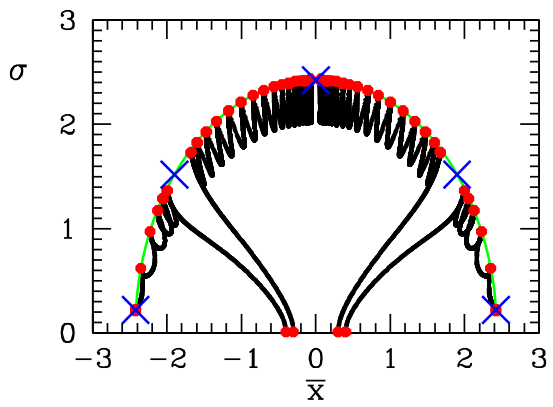

Figure 21. Iterates of the moment map seen in $(\bar{x}, \sigma)$ coordinates. Same as Figure 19 except over a larger range of coordinates, and same as Figure 14 except that here $\tau=1$.

increases (point 2). Projection back to Gaussian moves the system vertically downward to $\zeta=0$ (point 3 ).

Now consider the case $a_{1} \simeq 1$, near the local minima of the potential wells (we consider only the right well; the left is the same). One sees in Figures 17 and 20 that points on the slow manifold correspond to nearly Gaussian densities. This can be most clearly seen in Figure 20, where the slow manifold falls to near $\zeta=0$. The end point of the green curve is $a_{1}=1$. In Figure 17 we see that the red points (the Gaussian densities in $a$ coordinates) fall very nearly to $\chi=0$, meaning that these densities are almost exactly represented by a sum of $\phi_{0}$ and $\phi_{1}$. The small gap between the slow manifold and the Gaussian density (seen in both Figures 17 and 20) reflects the fact that the metastable density is not exactly Gaussian.

In Figure 21 we show the dynamics in $(\bar{x}, \sigma)$ coordinates, showing all the fixed points of the moment map. This is similar to Figure 14(right), except that here $\tau=1$. The figure shows trajectories starting from four initial conditions. Note that the slow manifold accurately captures the unstable manifold of the saddle fixed points.

4.4. Two-dimensional SDE. In this section we consider moment maps for the twodimensional SDE (2.4) presented in section 2.2. The dynamics are potentially richer than for the one-dimensional SDE considered up to now. Nevertheless, the stabilization of metastable states is the same as for the one-dimensional SDE.

As before, we examine maps for both first-order and second-order moments. The firstorder map can be written as $\Phi\left(\bar{x}_{1}, \bar{x}_{2}\right)$, where $\bar{x}_{1}$ and $\bar{x}_{2}$ are means of $Q$ and $P$, respectively. The second-order map can be written as $\Phi\left(\bar{x}_{1}, \bar{x}_{2}, \sigma_{1}, \sigma_{2}, c\right)$, where $\sigma_{1}$ and $\sigma_{2}$ are the standard deviations of $Q$ and $P$, respectively, and $c$ is the cross correlation. Other coordinates could be used for the five-dimensional phase space. We again consider the slightly asymmetric doublewell potential (2.3) for parameters similar to those used for the one-dimensional SDE. All results have been obtained numerically from simulations of (2.4) for the case $M=1, \gamma=1$, $\beta=2$.

Figure 22 shows typical phase portraits for the first-order and second-order moment maps. The first-order map exhibits the dynamics typical of bistable damped oscillators. Similar to the one-dimensional case, the fixed points are located at, or very close to, $\left(\bar{x}_{c}, 0\right)$, where $\bar{x}_{c}$ is a local extremum of the potential. The second-order map has five fixed points, similar to 

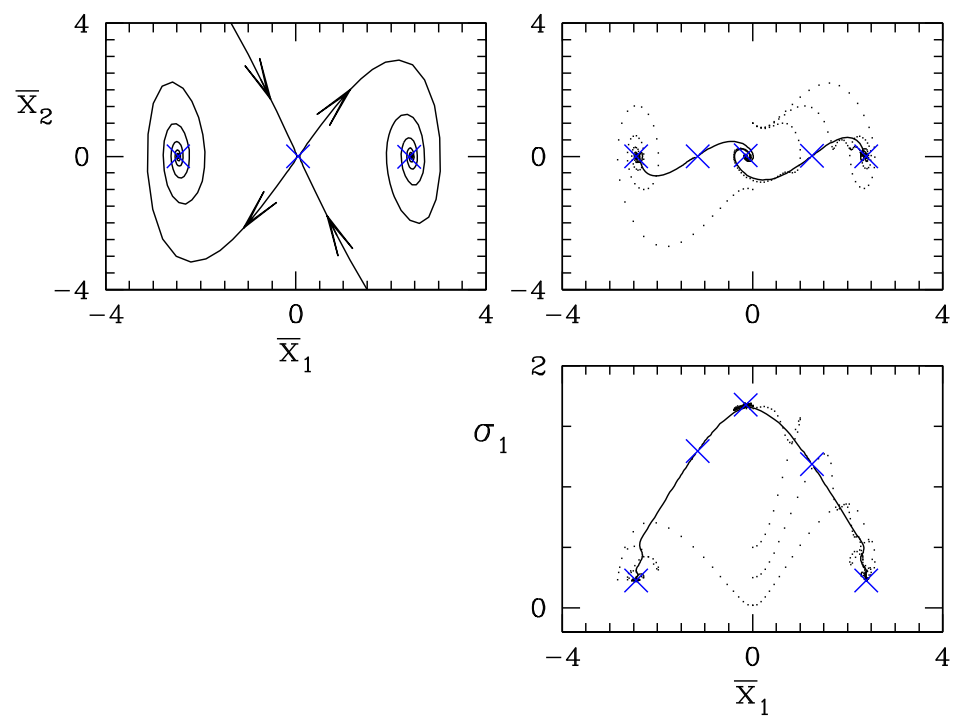

Figure 22. Phase portrait for first-order (left) and second-order (right) moment maps for the two-variable SDE with a slightly asymmetric double-well potential. Fixed points are indicated by crosses. For the first-order map, stable and unstable manifolds of the saddle fixed point are shown. For the second-order map, unstable manifolds of the saddle fixed point are shown. The stable manifolds are four-dimensional. Points show some representative trajectories. Parameters are $\mu=6, \nu=0.3, \tau=0.1$.

those seen in Figure 14, including a stable fixed point with a very broad Gaussian measure. Typically trajectories of interest approach either one or the other of the stable fixed points corresponding to the potential minima. The moment maps for the two-dimensional SDE stabilize metastable states in a very similar way to that found for the one-dimensional SDE.

Figure 23 provides a better view of the dynamics of the second-order moment map and shows how trajectories approach the stable fixed points. A Gaussian measure in two variables can be visualized as an ellipse in the plane specified by five numbers: the center, the semimajor and semiminor axes, and the orientation. Thus for each point in the five-dimensional phase space of the moment map we plot an ellipse in the plane. One can think of each ellipse as corresponding to a level set of a Gaussian density.

Two trajectories are shown, one with the ellipse at every iteration of the map plotted, and the other with only a few representative ellipses plotted. One can see how densities evolve under the map toward stable equilibria. Trajectories from almost all initial condition distributions that are not too broad (i.e., initialized within one of the two metastable wells, as discussed above) evolve to one or the other of the two fixed points, similarly to what is shown in Figure 23.

For completeness we show in Figure 24 bifurcation diagrams for the moment maps for the two-dimensional SDE. These bifurcation diagrams reveal much the same features as for the one-dimensional SDE. Fixed points of the first-order moment map track the potential minima. For the second-order map, stable fixed points corresponding to metastable states exist except in regions near where the potential bifurcates from single- to double-well. Clearly, close to such parameter values, the separation of time scales between equilibration in one well and 


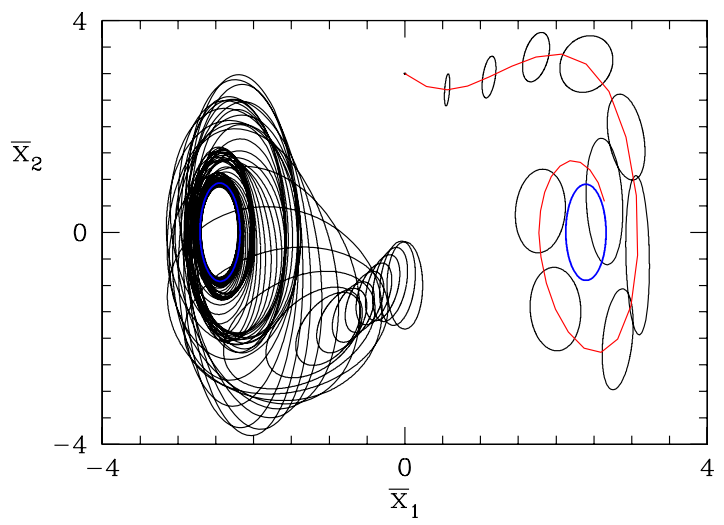

Figure 23. Dynamics of the second-order moment map for the two-variable SDE. Two trajectories are visualized by plotting ellipses corresponding to points in the five-dimensional phase space. One trajectory starts at $\left(\bar{x}_{1}, \bar{x}_{2}, \sigma_{1}, \sigma_{2}, c\right)=(0,3,0.01,0.01,0)$. Only a few representative ellipses are shown. The curve shows the path of the center of the ellipses. The other trajectory starts at $\left(\bar{x}_{1}, \bar{x}_{2}, \sigma_{1}, \sigma_{2}, c\right)=(0,-1,0.1,0.5,0)$. In this case every point on the trajectory is shown. Two stable fixed points of the map are shown as bold blue ellipses. Parameters are $\mu=6, \nu=0.3, \tau=0.1$. See also the accompanying animations (63866_04.gif [3.8MB] and 63866_05.gif [7.5MB]).
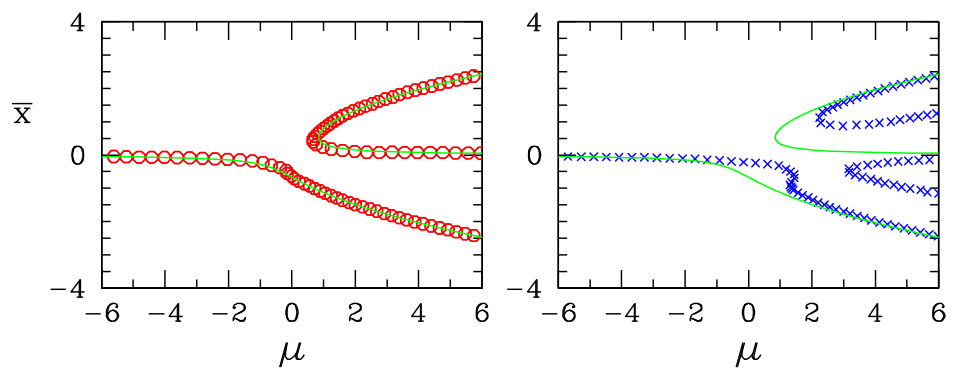

Figure 24. Bifurcation diagrams for the first-order (left) and second-order (right) moment maps for the two-variable SDE. Parameters are $\nu=0.3, \tau=0.1$.

transition to the other is no longer present, and the fixed points that we find depend on the map reporting horizon $\tau$. Fixed points, both stable and unstable, corresponding to broad distributions, separate the stable fixed points. While not shown, we find that the fixed points corresponding to the stabilized metastable states are essentially independent of the time $\tau$ over which the map is defined; this suggests that the map is a good effective description in their neighborhood, but not a useful one close to the $\tau$-dependent fixed points. The ability to test the sensitivity of the map dynamics and fixed points to the parameter $\tau$, as well as the ability to use maps of different orders, grants us potential tools with which to "test online" the validity of a given map as an effective model of the detailed dynamics.

4.5. Heat bath. Finally, we consider the dynamics of a particle in a heat bath as described in section 2.1. Moment maps for this example are of the type defined for ODE systems in section 3.2. Nevertheless we expect the moment map for the ODE system to behave very much 
like that of the two-dimensional SDE, since the SDE is known to capture the dynamics of the heat-bath model in the limit of a large bath. From a computational viewpoint, in fact, there is not much distinction between the stochastic (SDE) and the deterministic (ODE) cases, since in both cases we numerically compute the moment maps using Monte Carlo simulations to evolve densities over time interval $\tau$.

There are two related new issues, however. The first is that $N$, the number of particles in the heat bath, is now a parameter which could potentially affect the dynamics of the system. The other related issue is that there is a minimum time interval over which we should define the moment map. For any given bath size $N$ there is a maximum frequency $\omega_{m}$ of the oscillators in the bath, where $\omega_{m} \simeq N^{1 / 3}$. We therefore should take the map time $\tau$ to be at least of the same order as the $1 / \omega_{m}$. We always use $\tau>2 \pi / \omega_{m}$.

Other than the preceding two points, the only significant difference between the heat bath and the two-dimensional SDE is that the heat bath requires significantly more computation to evolve densities forward in time. Hence the moment map is much more expensive to compute for the heat bath than for the SDE. For this reason, we have limited our studies to a fixed potential: (2.3) with $\mu=4, \nu=0.3$. This is the potential used for the simulations shown in the introduction.

Figure 25 shows phase portraits of the moment map for the heat-bath model. The firstorder map shows the expected three fixed points at the local extrema of the potential and exhibits the dynamics of bistable damped oscillators. The map has indeed stabilized the metastable states with a well-defined boundary (the stable manifold of the saddle) separating the basins of attraction. The location of the fixed points does not depend in any significant way on the number of particles in the bath or on the map time $\tau$. The stable and unstable manifolds do vary somewhat, primarily with the map time $\tau$, but are always qualitatively as seen in Figure 25.

For the second-order map we show phase portraits in the style of Figure 23 for the twodimensional SDE system. We show two trajectories for each of three cases. The middle case is for $N=8000$, the value used for the simulations shown in the introduction. The maximum oscillator frequency in the bath is $\omega_{m} \simeq 20$, and so we use a map time of $\tau=0.4>\pi / 10$.

We show a map starting at $\left(\bar{x}_{1}, \bar{x}_{2}, \sigma_{1}, \sigma_{2}, c\right)=(0,3,0.01,0.01,0)$. This initial condition corresponds exactly to the initial density used Figure 3. One sees the similarity between the trajectory for the moment map and the evolution of the density in Figure 3. However, the "leaking" of mass into the left potential well observed in Figure 3 is prevented by the moment map via the mechanism illustrated in Figure 17. The moment map is nonlinear and has the two stable fixed points where the density is only metastable. Most trajectories evolve to one or the other of these fixed points.

For the same potential we have investigated the effect of $N$, the number of particles in the heat bath, on the moment map. We find that the map is quite insensitive to $N$, at least for $N \geq 1000$. For large $N$ one can use a smaller map time $\tau$, and the dynamics do depend weakly on $\tau$. Specifically, the fixed points for the three cases in Figure 25 are very slightly different, though this cannot be seen on the scale of the figure. This difference is not due directly to $N$ but to the value of $\tau$.

5. Conclusions. In this paper we have introduced a mathematical framework intended to outline and clarify some aspects of the coarse-grained approach to analyzing stochastic and 

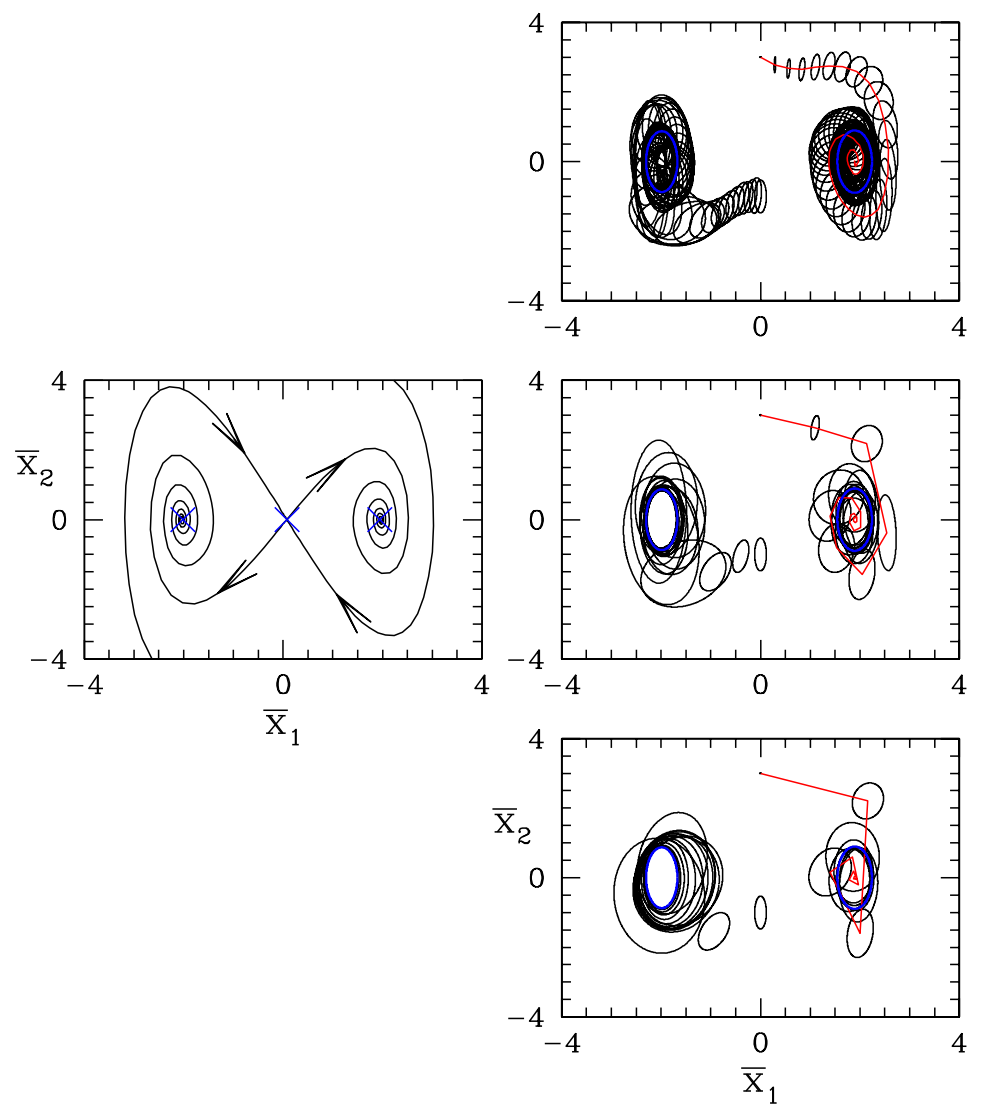

Figure 25. Phase portraits showing the dynamics of moment maps for the heat bath model. First-order (left) and second-order (right) maps are shown. For the first-order map $N=8000$ particles is used. For the second-order map the following are used: (top) $N=256000, \tau=0.1$; (middle) $N=8000, \tau=0.4$; (bottom) $N=1000, \tau=0.8$. Other parameters are $\mu=4$ and $\nu=0.3$.

deterministic systems. In particular, we have given a precise definition of the moment map. These are maps on the (low-dimensional) space of low-order moments of probability measures. We have considered these maps both for stochastic systems and for deterministic systems with random initial data. While the underlying evolution of densities in both systems is linear, the moment maps are typically nonlinear. Our main focus has been understanding the origins of this nonlinearity.

In this paper we sought to establish a connection between the dynamics of coarse-grained observables (such as moments of evolving realization ensembles) and the nonlinear dynamics one expects at the deterministic limit. Contemporary estimation techniques would allow us to recover both the deterministic and the stochastic components of an effective stochastic model (e.g., [1]). Then, instead of using integral changes of coarse-grained observables, we could directly seek the extrema of an underlying effective potential, or even the extrema of the corresponding equilibrium density (see, e.g., [18, 27, 39]).

We have presented results for a number of model systems. We have first considered the 
simple OU process, for which a full analysis is possible. Then, using a combination of numerical studies and analysis, we have considered in most detail a one-dimensional SDE with a doublewell potential. This system provides the simplest example of a nonlinear moment map. In particular, this example shows how the moment map can stabilize metastable densities of the underlying linear flow. We have additionally presented numerical results for moment maps computed for two other systems with double-well potentials - a two-dimensional SDE and a deterministic ODE system with many degrees of freedom. Maps for both of these systems show the basic features found for the one-dimensional SDE, namely, nonlinearity and the stabilization of metastable densities.

One of the important issue that arises naturally in this computational framework is the importance of the observer. How long does a physical observer have to wait before declaring that an observed quantity is at steady state? This is clearly related to our testing the dependence of the map fixed points on the map reporting horizon. We also saw that the initialization of computational experiments (whether within or outside a well) can be vital in the existence of an effective reduced model (this is related, as we mentioned, to conditional averaging techniques).

\section{REFERENCES}

[1] Y. Ait-Sahalia, Maximum-likelihood estimation of discretely sampled diffusions: A closed-form approximation approach, Econometrica, 70 (2002), pp. 223-262.

[2] A. Angeletti, C. Castagnari, and F. Zirilli, Asymptotic eigenvalue degeneracy for a class of onedimensional Fokker-Planck operators, J. Math. Phys., 26 (1985), pp. 678-691.

[3] A. Chorin, O. H. Hald, And R. Kupferman, Optimal prediction and the Mori-Zwanzig representation of irreversible processes, Proc. Natl. Acad. Sci. USA, 97 (2000), pp. 2968-2973.

[4] J. Cisternas, C. W. Gear, S. Levin, And I. G. Kevrekidis, Equation-free modeling of evolving diseases: Coarse-grained computations with individual-based models, Proc. R. Soc. Lond. Ser. A Math. Phys. Eng. Sci., 460 (2004), pp. 27621-2779; also available online at http://arXiv.org/abs/nlin.AO/ 0310011.

[5] P. Deuflhard, W. Huisinga, W. Fischer, and C. Schütte, Identification of almost invariant aggregates in reversible nearly uncoupled Markov chains, Linear Algebra Appl., 315 (2000), pp. 39-59.

[6] A. Doucet, N. de Freitas, and N. J. Gordon, Sequential Monte Carlo Methods in Practice, SpringerVerlag, New York, 2001.

[7] W. E, D. Liu, And E. Vanden-Eijnden, Analysis of multiscale methods for stochastic differential equations, Comm. Pure Appl. Math., 58 (2005), pp. 1544-1585.

[8] P. Ehrenfest, Collected Scientific Papers, North-Holland, Amsterdam, 1959.

[9] R. Erban, I. G. Kevrekidis, And H. G. Othmer, An equation-free computational approach for extracting population-level behavior from individual-based models of biological dispersal, Phys. D, 215 (2006), pp. 1-24.

[10] G. W. Ford ANd M. Kac, On the quantum Langevin equation, J. Statist. Phys., 46 (1987), pp. 803-810.

[11] C. W. Gardiner, Handbook of Stochastic Methods, 2nd ed., Springer-Verlag, New York, Berlin, 1985.

[12] C. W. Gear, I. G. Kevrekidis, And K. Theodoropoulos, "Coarse" integration/bifurcation analysis via microscopic simulators: Micro-Galerkin methods, Comp. Chem. Engrg., 26 (2002), pp. 941-963.

[13] C. W. Gear And I. G. Kevrekidis, Projective methods for stiff differential equations: Problems with gaps in their eigenvalue spectrum, SIAM J. Sci. Comput., 24 (2003), pp. 1091-1106.

[14] C. W. Gear and I. G. KevreKidis, Telescopic projective integrators for stiff differential equations, J. Comput. Phys., 187 (2003), pp. 95-109.

[15] C. W. GEAR, Projective Integration Methods for Distributions, Technical Report NEC TR 2001130, NEC Institute, Princeton, NJ, 2001. 
[16] D. Givon, R. Kupferman, and A. M. Stuart, Extracting macroscopic dynamics: Model problems and algorithms, Nonlinearity, 17 (2004), pp. R55-R127.

[17] A. N. Gorban, I. V. Karlin, H. C. Oettinger, and L. L. Tatarinova, Ehrenfest's argument extended to a formalism of nonequilibrium thermodynamics, Phys. Rev. E (3), 63 (2001), paper 066124.

[18] M. Hahtaja, D. Srolovitz, and I. G. Kevrekidis, Apparent hysteresis in a driven system with selforganized drag, Phys. Rev. Lett., 92 (2004), paper 160603; also available online at http://arXiv.org/ abs/cond-mat/0310460.

[19] O. HALD, Optimal prediction of the Klein-Gordon equation, Proc. Natl. Acad. Sci. USA, 96 (1999), pp. 4774-4779.

[20] C. Hillermeier, N. Kunstmann, B. Rabus, and P. Tavan, Topological feature maps with self-organized lateral connections: A population coded, one-layer model of associative memory, Biol. Cybernet., 72 (1994), pp. 103-117.

[21] W. Huisinga, Metastability of Markovian Systems, Ph.D. thesis, Mathematics Department, Free University, Berlin, 2003; available online at http://page.mi.fu-berlin.de/ huisinga/publications/index.html.

[22] G. Hummer and I. G. Kevrekidis, Coarse molecular dynamics of a peptide fragment: Free energy, kinetics and long time dynamics computations, J. Chem. Phys., 118 (2003), pp. 10762-10773; also available online at http://arXiv.org/abs/physics/0212108.

[23] C. T. Kelley, I. G. Kevrekidis, And L. QiaO, Newton-Krylov solvers for time-steppers, SIAM J. Appl. Dyn. Syst., submitted; also available online at http://arXiv.org/abs/math.DS/0404374.

[24] C. T. Kelley, Iterative Methods for Linear and Nonlinear Equations, Frontiers Appl. Math. 16, SIAM, Philadelphia, 1995.

[25] I. G. Kevrekidis, C. W. Gear, J. M. Hyman, P. G. Kevrekidis, O. Runborg, and K. TheodorOPOULOS, Equation-free coarse-grained multiscale computation: Enabling microscopic simulators to perform system-level tasks, Commun. Math. Sci., 1 (2003), pp. 715-762; original version available online at http://arXiv.org/abs/physics/0209043.

[26] I. G. Kevrekidis, C. W. Gear, And G. Hummer, Equation-free: The computer-assisted analysis of complex, multiscale systems, AIChE J., 50 (2004), pp. 1346-1354.

[27] D. I. Kopelevich, A. Z. Panagiotopoulos, and I. G. KevreKidis, Coarse-grained kinetic computations of rare events: Application to micelle formation, J. Chem. Phys., 122 (2005), paper 044908; also available online at http://arXiv.org/abs/cond-mat/0407220.

[28] R. Kupferman, A. M. Stuart, J. Terry, And P. Tupper, Long time behaviour of large mechanical systems with random initial data, Stoch. Dyn., 2 (2002), pp. 533-562.

[29] A. Makeev, D. Maroudas, And I. G. Kevrekidis, "Coarse" stability and bifurcation analysis using stochastic simulators: Kinetic Monte Carlo examples, J. Chem. Phys., 116 (2002), pp. 10083-10091.

[30] A. G. Makeev, D. Maroudas, A. Z. Panagiotopoulos, And I. G. Kevrekidis, Coarse bifurcation analysis of kinetic Monte Carlo simulations: A lattice gas model with lateral interactions, J. Chem. Phys., 117 (2002), pp. 8229-8240.

[31] A. G. Makeev And I. G. Kevrekidis, Equation-free multiscale computations for a lattice-gas model: Coarse-grained bifurcation analysis of the $\mathrm{NO}+\mathrm{CO}$ reaction on Pt(100), Chem. Eng. Sci., 59 (2004), pp. 1733-1743.

[32] B. J. Matkowsky And Z. Schuss, Eigenvalues of the Fokker-Planck operator and the approach to equilibrium for diffusions in potential fields, SIAM J. Appl. Math., 40 (1981), pp. 242-254.

[33] E. Nelson, Dynamical Theories of Brownian Motion; available online at http://www.math.princeton. edu/ nelson/books.html.

[34] G. Pavliotis And A. M. Stuart, White noise limits for inertial particles in a random field, Multiscale Model. Simul., 1 (2003), pp. 527-553.

[35] R. Rico-Martinez, C. W. Gear, And I. G. Kevrekidis, Coarse projective kMC integration: Forward/ reverse initial and boundary value problems, J. Comput. Phys., 196 (2004), pp. 474-489; also available online at http://arXiv.org/abs/nlin.CG/0307016.

[36] C. Schuette, J. Walter, C. Hartmann, and W. Huisinga, An averaging principle for fast degrees of freedom exhibiting long-term correlations, Multiscale Model. Simul., 2 (2004), pp. 501-526.

[37] S. Setayeshgar, C. W. Gear, H. G. Othmer, and I. G. Kevrekidis, Application of coarse integration to bacterial chemotaxis, Multiscale Model. Simul., 4 (2005), pp. 307-327; also available online at http://arXiv.org/abs/physics/0308040. 
[38] C. Siettos, M. D. Graham, and I. G. Kevrekidis, Coarse Brownian dynamics for nematic liquid crystals: Bifurcation, projective integration and control via stochastic simulation, J. Chem. Phys., 118 (2003), pp. 10149-10157; also available online at http://arXiv.org/abs/cond-mat/0211455.

[39] S. Sriraman, I. G. Kevrekidis, and G. Hummer, Coarse nonlinear dynamics of filling-emptying transitions: Water in carbon nanotubes, Phys. Rev. Lett., 95 (2005), paper 130603; also available online at http://arXiv.org/abs/cond-mat/0503491.

[40] K. Theodoropoulos, Y.-H. QIan, And I. G. Kevrekidis, "Coarse" stability and bifurcation analysis using timesteppers: A reaction diffusion example, Proc. Natl. Acad. Sci. USA, 97 (2000), pp. 98409843.

[41] K. Theodoropoulos, K. Sankaranarayanan, S. Sundaresan, and I. G. Kevrekidis, Coarse bifurcation studies of bubble flow lattice Boltzmann simulations, Chem. Eng. Sci., 59 (2004), pp. 2357-2362; also available online at http://arXiv.org/abs/nlin.PS/0111040.

[42] E. VANDEn EIJNDEn, Numerical techniques for multi-scale dynamical systems with stochastic effects, Commun. Math. Sci., 1 (2003), pp. 377-384.

[43] R. Zwanzig, Nonlinear generalized Langevin equations, J. Statist. Phys., 9 (1973), pp. 215-220. 